\title{
Das Input-Output-Modell als allgemeiner Ansatz für die Produktionsfunktion der Unternehmung
}

\section{The Input-Output-Model as a General Production Function of the Firm}

\author{
Von Hans-Ulrich $\underbrace{\mathrm{K} u ̈ p p e r}$, Tübingen \\ 1. Übertragung des makroökonomischen Input-Out- \\ put-Modells auf den Produktionsprozeß der Unter- \\ neh m ung
}

Kennzeichnend für die neuere Entwicklung der betriebwirtschaftlichen Produktionstheorie sind die Gliederung des Produktionsprozesses der Unternehmung in Teilprozesse und die nähere Analyse der Beziehungen zwischen Realgütereinsatz und Realgüterausbringung dieser Teilprozesse ${ }^{1}$ ). Die Produktionsfunktion der Unternehmung wird durch eine Aggregation der Input-Output-Beziehungen aller Teilprozesse hergeleitet $\left.{ }^{2}\right)$. Hierzu ist es notwendig, die Verflechtungen zwischen den Teilprozessen $\mathrm{zu}$ erfassen. Für die Abbildung dieser strukturellen Beziehungen stellt der Input-Output-Ansatz eine geeignete Grundlage dar. Deshalb ist das makroökonomische Input-Output-Modell auf den einzelwirtschaftlichen Produktionsprozeß übertragen und durch die Berücksichtigung unterschiedlicher InputOutput-Funktionen der Teilprozesse erweitert worden ${ }^{3}$ ).

Der Produktionsprozeß einer Unternehmung setzt sich aus einer Vielzahl von Teilprozessen zusammen. Um zu einer präzisen Abbildung zu gelangen, die möglichst viele Typen von Produktionsprozessen umfaßt, erscheint es zweckmäßig, diese Teilprozesse und nicht Produktionsstellen als

$\left.{ }^{1}\right)$ G u t e n ber g (Produktion) $298 \mathrm{ff}$; K K l g e r (Produktionstheorie) $53 \mathrm{ff}$; $\mathrm{H}$ e in e n (Kostenlehre) $188 \mathrm{ff}$; $\mathrm{L} \mathrm{a} \mathrm{Bm}$ a n n (Erlösrechnung) $72 \mathrm{ff}$.; W it t m a n n (Produktionstheorie) $102 \mathrm{ff}$; $\mathrm{K} 1$ o o ck (Input-Output-Modelle) $39 \mathrm{ff}$; L ü cke (Produktionstheorie) $60 \mathrm{ff}$; $\mathrm{Press}$ mar (Leistungsanalyse) $118 \mathrm{ff}$; Franke (Betriebsmodelle) $33 \mathrm{ff}$;; $\mathrm{S}$ teff e n (Elementarfaktoren) $28 \mathrm{ff}$.; A d a m (Kostentheorie) $12 \mathrm{ff}$; $\mathrm{Schweitzer/K} \ddot{\mathrm{c} p \mathrm{per}}$ (Produktionstheorie) $87 \mathrm{ff}$.

$\left.{ }^{2}\right) \mathrm{K}$ l o o c k (Input-Output-Modelle) $126 \mathrm{ff}$.

3) $\mathrm{S} \mathrm{ch} \mathrm{u} \mathrm{man} \mathrm{n} \mathrm{(Input-Output-Analyse)} 286 \mathrm{ff}$.; K l o o ck (Input-OutputModelle) $66 \mathrm{ff}$; Voge 1 (Strukturbilanzen) $11 \mathrm{ff}$;; S chweitzer / K ü p per (Produktionstheorie) $46 \mathrm{ff}$. 
kleinste Einheiten des Input-Output-Ansatzes $\mathrm{zu}$ wählen ${ }^{4}$ ). Hierdurch werden auch die Fälle berücksichtigt, in denen dieselbe Produktionsstelle verschiedene Güterarten bearbeitet. Jeder Teilprozeß kann durch die in ihm erbrachte(n) materielle(n) oder immaterielle(n) Ausbringungsgüterar$t(e n)$ gekennzeichnet werden. Originäre Einsatzgüter wie Roh- und Betriebsstoffe, menschliche und maschinelle Arbeit lassen sich als Ausbringungsgüter von Beschaffungs- oder Bereitstellungsprozessen interpretieren $^{5}$ ). Damit gelingt es, sämtliche eingesetzten und erstellten Güterarten als Outputgüter aufzufassen. Ferner ist die Gliederung der Güter- und der Prozeßarten so vorzunehmen, daß jeder Prozeßart genau eine Güterart zugeordnet ist und umgekehrt. Dies erfordert einerseits eine Unterscheidung zwischen Gütern mit gleichen Eigenschaften, die in verschiedenartigen Teilprozessen erstellt werden. Andererseits sind bei der Erzeugung verschiedener Güterarten in einem $\mathrm{Ku}$ p pelprozeß eine Güterart als „Hauptprodukt“ $\mathrm{zu}$ definieren und für die im gleichen Prozeß anfallenden „Nebenprodukte“ fiktive Teilprozesse einzuführen.

Die in einem Teilprozeß erstellten Güter können (1) in anderen Teilprozessen der Unternehmung wiedereingesetzt, (2) am Markt abgesetzt oder (3) auf Lager gelegt werden. Bezeichnet man die Ausbringungsmenge des i-ten Teilprozesses mit $r_{i}$, ihre Teilmenge zum Einsatz im j-ten Prozeß mit $r_{i j}$ sowie die zum Absatz mit $x_{i}$ und die zur Lagerbestandsänderung ${ }^{6}$ ) mit $\Delta \mathrm{l}_{\mathrm{i}}$, dann gilt für jede Güterart die Beziehung:

$$
\mathrm{r}_{\mathrm{i}}=\sum_{\mathrm{j}} \mathrm{r}_{\mathrm{ij}}+\mathrm{x}_{\mathrm{i}}+\Delta \mathrm{l}_{\mathrm{i}}
$$

Die Gesamtheit der zwischen allen $\mathrm{i}=1, \ldots, \mathrm{n}$ Teilprozessen sowie von der Unternehmung zu ihrer Umwelt fließenden Realgüter kann durch folgendes Gleichungssystem abgebildet werden:

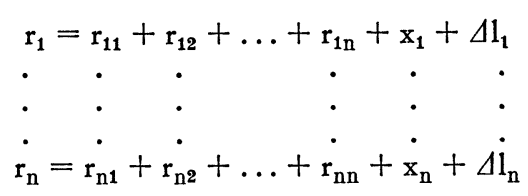

bzw. in Matrixschreibweise

$$
\mathrm{r}=\mathrm{R} \cdot \mathrm{e}+\mathrm{x}+\Delta 1
$$

Dabei sind $r, x$ und $\Delta l$ die Vektoren der Ausbringungsmengen, der Absatzmengen und der Lagerbestandsänderungen, während $\mathrm{e}^{\prime}=(1,1, \ldots, 1)$ den summierenden Vektor angibt. $26 \mathrm{ff}$

4) Vgl. auch Koopmans (Production) 35 f;; Bohr (Produktionstheorie)

$\left.{ }^{5}\right) \mathrm{K}$ l o o c k (Input-Output-Modelle) 52.

6) Vgl. hierzu Schweitzer (Kostentheorie) 23 ff. Eine Lagerbestandsabnahme ist doppelt begrenzt: Ihr absoluter Wert darf nicht größer sein als a) der vorhandene Lagerbestand und b) die Summe der Absatz- und Wiedereinsatzmengen dieser Güterart, da negative Ausbringungsmengen unzulässig sind. 
Bei statischer Betrachtung beziehen sich alle Gütermengen auf dieselbe Produktionsperiode. Von dieser Betrachtung wird im folgenden stets ausgegangen. In der $G \ddot{u}$ terflu $B \mathrm{matrix} R$ gilt jede Zeile für eine bestimmte Güterart und jede Spalte für eine bestimmte Prozeßart. Eine Zeile von $\mathbf{R}$ gibt an, welche Mengen einer Güterart in den verschiedenen Prozessen während der Produktionsperiode eingesetzt werden. Dagegen ist aus einer Spalte von $\mathbf{R}$ ersichtlich, welche verschiedenen Güterarten in einer Prozeßart eingesetzt werden. $\mathrm{Da}$ jede Güterart als Ausbringungsgut genau einer Prozeßart definiert wird, ist $\mathrm{R}$ quadratisch. Berücksichtigt man, welche Elemente von $\mathrm{R}$ ungleich Null sind, so werden die strukturellen Verflechtungen zwischen den Teilprozessen erkennbar. Die Analyse dieser Beziehungen kann mit Hilfe einer $S \mathrm{trukturmatrix} S$ durchgeführt werden, deren Elemente $s_{i j}$ wie folgt definiert $\left.\operatorname{sind}^{7}\right)$ :

$s_{i j}=\left\{\begin{array}{c}+1, \text { wenn die i-te Güterart im j-ten Teilprozeß direkt eingesetzt wird } \\ -1, \text { wenn die i-te Güterart im j-ten Teilprozeß als Nebenprodukt anfällt } \\ 0, \text { sonst }\end{array}\right.$

Die Strukturmatrix $S$ hat dieselbe Zeilen- und Spaltenzahl wie die Güterflußmatrix $R$ und weist an denselben Stellen Nullelemente auf wie diese. Sie bildet die Existenz direkter Beziehungen zwischen den Teilprozessen $a b$, während $R$ die Quantität dieser direkten Beziehungen wiedergibt.

Für die Herleitung der Produktionsfunktion einer Unternehmung sind neben der Produktionsstruktur Hypothesen über die Input-Output-Beziehungen der einzelnen Teilprozesse maßgebend. Diese Hypothesen können Transformationsf unktionen genannt werden ${ }^{8}$ ). Jede Transformationsfunktion bildet für einen Teilprozeß $\mathrm{j}$ des gesamten Produktionsprozesses die quantitativen Beziehungen zwischen den Einsatzgütermengen $r_{i j}$, der Ausbringungsgütermenge $r_{j}$ und gegebenenfalls sonstigen Einflußgrößen $e_{j}{ }^{1}, \ldots, e_{j}{ }^{z}$ ab. Bei impliziter Schreibweise lautet sie allgemein:

$$
f_{j}\left(r_{j}, r_{1 j}, \ldots, r_{n j}, e_{j}{ }^{1}, \ldots, e_{j}^{z}\right)=0
$$

Die Transformationsfunktion läßt sich so umformen, daß man für jede Prozeßart j ein System aus Gleichungen der Art

$$
r_{i j}=f_{i j}\left(r_{1 j}, \ldots, r_{i-1, j}, r_{i+1, j}, \ldots, r_{n j}, e_{j}^{1}, \ldots, e_{j}^{z}, r_{j}\right) \cdot r_{j}
$$

erhäl $\left.t^{9}\right)$. In dieser Form $r_{i j}=f_{i j}(\ldots) \cdot r_{j}$ kann man die Transformationsfunktionen in das Gleichungssystem (1) einsetzen. Es ergibt sich allgemein:

${ }^{7}$ ) Vgl. C z a y k a (Input-Output-Analyse) 1; K lo o c k (Input-Output-Modelle) $52 \mathrm{ff}$. Die Erfassung von Kuppelprodukten als "negative Inputs" schlägt Felth a vor. Felth am (Approaches) $13 \mathrm{ff}$.; vgl. auch Butterw or th/Sigl o c h (Input-Output-Model) $709 \mathrm{ff}$. und Abschnitt $4 \mathrm{c}$.

8) K 1 o o c k (Input-Output-Modelle) 44.

9) K loo k (Input-Output-Modelle) $44 \mathrm{ff}$; K 1 o o ck (Input-Output-Analyse) $1955 \mathrm{ff}$. 


$$
\begin{aligned}
& r_{1}=f_{11}(\ldots) \cdot r_{1}+f_{12}(\ldots) \cdot r_{2}+\ldots+f_{1 n}(\ldots) \cdot r_{n}+x_{1}+\Delta l_{1} \\
& \begin{array}{llllll}
\cdot & \cdot & \cdot & \cdot & \cdot & \cdot
\end{array} \\
& \dot{r}_{n}=\dot{f}_{n 1}(\ldots) \cdot \dot{r}_{1}+\dot{f}_{n 2}(\ldots) \cdot r_{2}+\ldots+\dot{f}_{n n}(\ldots) \cdot r_{n}+\dot{x}_{n}+\dot{\Delta l_{n}}
\end{aligned}
$$

Faßt man die in den Transformationsfunktionen (2) enthaltenen direkten funktionalen Beziehungen $f_{i j}(\ldots)$ zwischen den Einsatzmengen $r_{i j}$ und den Ausbringungsmengen $r_{j}$ der Teilprozesse in einer Direkt brauchsmatrix F zusammen, so erhält man in Matrixschreibweise das Input-Output-Modell der Unternehmung ${ }^{10}$ ).

$$
\mathrm{r}=\mathrm{F} \cdot \mathrm{r}+\mathrm{x}+\Delta \mathrm{l}
$$

Durch Umformung gelangt man zu:

$$
\mathrm{r}=(\mathrm{E}-\mathrm{F})^{-1} \cdot(\mathrm{x}+\Delta \mathrm{l})
$$

Hierbei ist die Gesamtverbrauchsmatrix (E-F)-1 zu bestimmen, die neben den direkten auch die indirekten funktionalen Beziehungen zwischen den Teilprozessen enthält. Der Ausbringungsvektor $r$ umfaßt einerseits die originären Einsatzgütermengen als Ausbringungsmengen von Beschaffungsprozessen und andererseits die Mengen der in der Unternehmung hergestellten Güterarten. Wenn die Beschaffungsprozesse als erste $\mathrm{m}$ Teilprozesse des gesamten Produktionsprozesses numeriert werden, geben die ersten $m$ Gleichungen von (4)

$$
r_{m}=(E-F)_{m}^{-1} \cdot(x+\Delta l)
$$

die quantitativen Beziehungen zwischen originärem Gütereinsatz und den Absatzmengen sowie Lagerbestandsänderungen der Unternehmung wieder. Sie stellen einen allgemeinen Ansatz für die Produktionsfunktion der Unternehmung dar. Aus diesem können durch spezielle Hypothesen über die Verflechtungen $z$ wischen den Teilprozessen und die Art der Transformationsfunktionen Typen von Produktionsfunktionen für bestimmte Anwendungsbedingungen hergeleitet werden. Wesentliche Komponenten sind dabei die Produktionsstruktur und die Transformationsfunktionen.

\section{Abbildungder Produktionsstruktur}

a) Zerlegung der Strukturmatrix

Die Ausprägung der Produktionsstruktur läßt sich mit Hilfe der Strukturmatrix $S$ kennzeichnen. Hierzu ist es einmal zweckmäßig, die Strukturmatrix nach wichtigen Klassen von Güter- und Prozeßarten in Teilmatrizen $\mathrm{zu}$ zerlegen. Zum andern ist $\mathrm{zu}$ untersuchen, zwischen welchen Güterbzw. Prozeßarten direkte Beziehungen bestehen. Für alle Güter- und Pro-

${ }^{10}$ ) K l o ock (Input-Output-Modelle) $101 \mathrm{ff}$; S c hweitzer/Kü p per (Produktionstheorie) $46 \mathrm{ff}$. 
zeßarten, zwischen denen keine direkten Beziehungen vorliegen, wird die entsprechende Teilmatrix aus $S$ zur Nullmatrix. Darüber hinaus kann die Struktur der einzelnen Teilmatrizen von $S$ näher analysiert werden.

Als wichtige Klassen betrieblicher Realgüterarten können menschliche und maschinelle Arbeit, Betriebsstoffe, Rohstoffe und die in der Unternehmung erstellten $\mathrm{Zwischen-}$ und Endprodukte unterschieden werden ${ }^{\mathbf{1 1}}$ ). Unterstellt man, daß menschliche und maschinelle Arbeit als Leistungsabgaben von Produktiveinheiten erbracht werden, und bezeichnet man die Güterarten durch die Indices R für Rohstoffe, B für Betriebsstoffe, M für Leistungsabgaben von Produktiveinheiten sowie $P$ für $Z$ wischen- und Endprodukte, so ergibt sich die zerlegte Strukturmatrix $\mathrm{S}^{*}$ :

$$
s^{*}=\left[\begin{array}{llll}
s_{R R} & s_{R B} & s_{R M} & s_{R P} \\
s_{B R} & s_{B B} & s_{B M} & s_{B P} \\
s_{H R} & s_{H B} & s_{M M} & s_{M P} \\
s_{P R} & s_{P B} & s_{P M} & s_{P P}
\end{array}\right]
$$

Durch die getrennte Erfassung von menschlicher und maschineller Arbeit sowie eine stärkere Differenzierung sämtlicher Güterarten (z.B. der Betriebsstoffe nach Treib- und Brennstoffen, Strom und Kleinwerkzeugen $^{12}$ ), der Produkte nach Haupt-, Neben- und Hilfsprodukten usw.) und Prozeßarten (z.B. Prozesse für Markterkundung, Bestellabwicklung, Materialprüfung und Transport im Beschaffungsbereich oder Versand, Werbung und Kundendienst im Absatzbereich usw.) läßt sich der Präzisionsgrad der Analyse wesentlich erhöhen. Um die Fruchtbarkeit des Input-Output-Ansatzes für die Abbildung produktionstheoretischer Zusammenhänge grundsätzlich zu verdeutlichen, kann im folgenden von der vereinfachten zerlegten Strukturmatrix $S *(5)$ ausgegangen werden.

\section{b) Auftretenzyklischer Verflechtungen}

Ein grundlegendes Merkmal zur Kennzeichnung der Produktionsstruktur besteht in der Existenz von $\mathrm{Zyk} \mathrm{len}$ oder Schleifen ${ }^{13}$ ). Ein Zyklus liegt vor, wenn eine Güterart i direkt oder indirekt zur Herstellung einer Güterart $j$ und die Güterart $j$ direkt oder indirekt zur Erzeugung der Güterart $i$ eingesetzt werden. Dann kann die Strukturmatrix $S$ nicht auf die Form einer Dreicksmatrix gebracht werden. Beim Auftreten von Produktionszyklen lassen sich die Einsatzmengen für ein gegebenes $\mathrm{Ab}$ satz- und Lagerbestandsänderungsprogramm nur simultan für mehrere oder alle Prozeßarten der Unternehmung bestimmen. Produktionszyklen

$\left.{ }^{11}\right) \mathrm{Vgl}$. G u t e n b e r g (Produktion) 2 ff.

12) Vgl. He in e n (Kostenlehre) 190.

13) Vgl. K 1 o o c k (Input-Output-Modelle) $55 \mathrm{ff}$. 
kommen insbesondere vor, wenn Einsatzgüter als Hilfsprodukte selbst erstellt und bei der Erzeugung dieser Güter Zwischen- oder Endprodukte der Unternehmung verbraucht werden. Sie dürfen keine negativen Elemente in der Gesamtverbrauchsmatrix $(\mathrm{E}-\mathrm{F})^{-1}$ und keine negativen Einsatzgütermengen zur Folge haben. Diese Bedingung beinhaltet, daß die zur Erzeugung einer Einheit des Hilfsprodukts erforderliche Menge eines Zwischen- oder Endprodukts (deutlich) kleiner als eine Einheit ist.

\section{c) Mehrstufigkeit der Stückprozesse}

Wichtige Merkmale zur Kennzeichnung industrieller Produktionsprozesse sind die Mehrstufigkeit und die Vergenz der Stückprozesse. Als kleinsten Teilprozeß der Fertigung kann man entsprechend dem Vorgehen in der Praxis den A rbeitsgang verwenden. Hierunter versteht man einen raum-zeitlich abgeschlossenen Arbeitsprozeß, der von einer Person oder Personengruppe an einem Werkstoff unter Verwendung von Arbeitsmitteln vollzogen wird ${ }^{14}$ ). Die Menge der zur Herstellung einer Endproduktart durchzuführenden Arbeitsgänge bildet deren $S \mathrm{tückprozeB^{15 }}$ ). Bei mehrstufigen Stückprozessen müssen nacheinander zwei oder mehr Arbeitsgänge vollzogen werden.

Definiert man für jeden Arbeitsgang eine eigene ( $Z$ wischen- oder End-)Produktart, so ist die Teilmatrix $S_{\mathrm{PP}}$ bei mehrstufigen Stückprozessen keine Nullmatrix. Dagegen wird bei einstufiger Fertigung in jedem Arbeitsgang eine Endproduktart erzeugt. Dann bestehen keine Verflechtungen $z$ wischen verschiedenen Arbeitsgängen der Fertigung. Einstufige Stückprozesse sind somit daran erkennbar, daß $S_{P P}$ nur Nullelemente enthält. Jedoch ist bei industriellen Unternehmungen die Teilmatrix $S_{R P}$ mit positiven Elementen besetzt, während bei Handelsunternehmungen auch $S_{R P}$ eine Nullmatrix darstellt.

\section{d) Vergenz derStückprozesse}

Durch das Merkmal der Vergenz wird der Fluß der Werkstoffe beschrieben, zu denen neben $Z$ wischen- und Endprodukten auch die Rohstoffe gerechnet werden ${ }^{16}$ ). Deshalb ist für die Analyse dieses Merkmals zusätzlich zur Produktverflechtungsmatrix $S_{\mathrm{PP}}$ die Rohstoffeinsatzmatrix $S_{R P}$ zu berücksichtigen. Als wichtigste Vergenztypen lassen sich glatte (durchgängige), konvergierende (synthetische), programmbedingt divergierende, prozeßbedingt divergierende (analytische) und umgruppie-

14) Kosiol (Organisation) $196 \mathrm{f}$; REFA (Arbeitsstudium 1) 76; $\mathrm{Sch}$ weitzer/Kü p p e r (Produktionstheorie) 156. Eine Zerlegung von Arbeitsgängen bis zu Vorgangselementen ist möglich und kann entsprechend dem Vorschlag von $\mathrm{He}$ in en als Bildung von Elementarkombinationen aufgefaßt werden. Vgl. H e in e n (Kostenlehre) $220 \mathrm{ff}$.

$\left.{ }^{15}\right) \mathrm{S} \mathrm{ch}$ we it z e r (Probleme) 12.

$\left.{ }^{16}\right) \mathrm{G}$ u te n berg (Produktion) $4 \mathrm{f}$. 
rende Materialflüsse unterscheiden ${ }^{17}$ ). Bei einem glat t e n Stückprozeß wird in jedem Teilprozeß lediglich eine Werkstoffart eingesetzt und eine Werkstoffart erzeugt. Deshalb kann in der Teilmatrix der Rohstoffeinsatzmatrix $\mathrm{S}_{\mathrm{RP}}{ }^{18}$ ) sowie der Produktverflechtungsmatrix $\mathrm{S}_{\mathrm{PP}}$, die einen Stückprozeß abbildet, in jeder Zeile und jeder Spalte höchstens ein Element gleich eins sein (vgl. Abbildung 1).
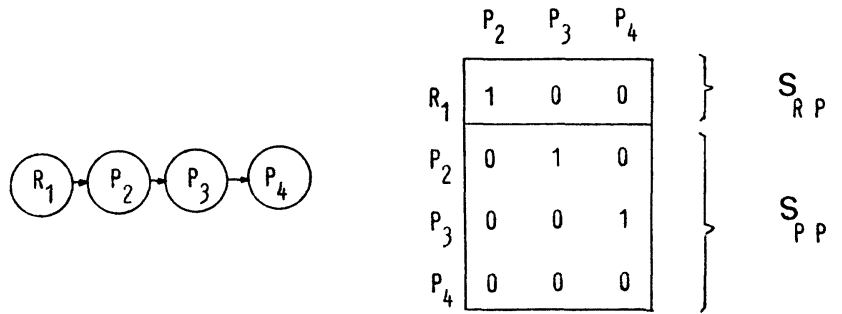

Abb. 1: Beispiel für Graph und Strukturmatrix eines glatten Stückprozesses.

Wird eine Produktart durch die Vereinigung mehrerer Werkstoffarten erzeugt, so liegt ein konvergierender Teilprozeß vor. Derartige Prozesse sind für die Montage mehrteiliger Produkte und für chemische Prozesse typisch. Es gibt also Arbeitsgänge, in denen mehrere Werkstoffarten zur Herstellung einer Güterart eingesetzt werden. Faßt man die Rohstoffeinsatzmatrix $S_{R P}$ und die Produktverflechtungsmatrix $S_{P P} z u-$ sammen, so sind in mindestens einer Spalte der sich ergebenden Matrix zwei Elemente gleich eins (vgl. Abbildung 2).
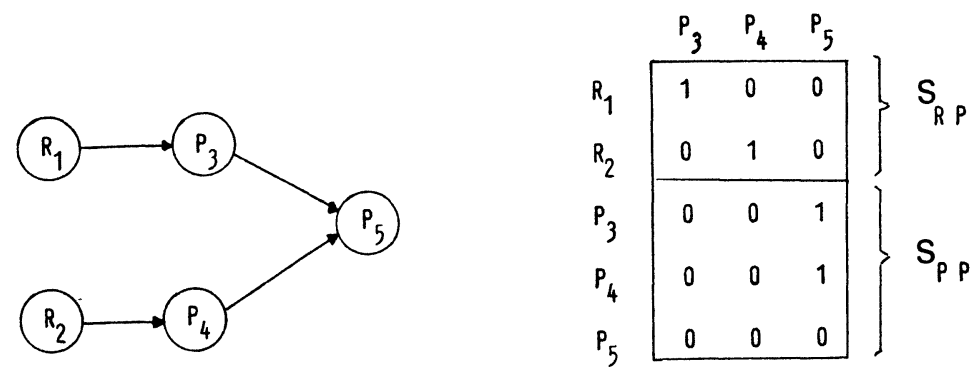

Abb. 2: Beispiel für Graph und Strukturmatrix eines konvergierenden Stückprozesses.

${ }^{17}$ ) $\mathrm{Zu}$ diesen Typen vgl. Ko siol (Aktionszentrum) 168; R i e be 1 (Erzeugungsverfahren) $55 \mathrm{ff}$;; $\mathrm{S} \mathrm{chäfer}$ (Industriebetrieb) $19 \mathrm{ff}$;; Große-O e $\mathrm{tr}$ in g h a us (Fertigungstypologie) $169 \mathrm{ff}$.

18) Man kann glatte Prozesse so definieren, daß $Z$ wischenprodukte jeweils nur zu einer Endproduktart, Rohstoffe aber zu verschiedenen Endproduktarten weiterverarbeitet werden (können). Dann können in $S_{R P}$ für verschiedene Stückprozesse mehrere Einserelemente je Zeile auftreten. 
Treten in der Produktverflechtungsmatrix $S_{\mathrm{PP}}$ in einer Zeile mehrere positive Einserelemente auf, so wird eine $Z$ wischenproduktart zur Herstellung verschiedener Produktarten verwendet. Die gesamte Herstellungsmenge einer Güterart wird auf mehrere, im Produktionsprogramm enthaltene Produktarten aufgeteilt. Man kann diesen Vergenztyp daher als programmbedingte Divergenz bezeichnen ${ }^{19}$ ) (vgl. Abbildung $3)$.
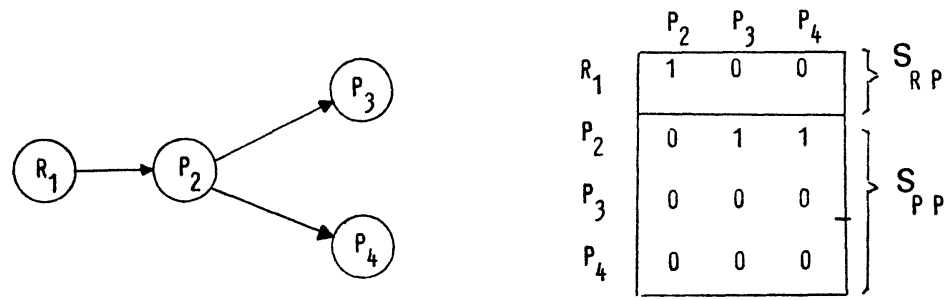

Abb. 3: Beispiel für Graph und Strukturmatrix eines programmbedingt divergierenden Stückprozesses.

Weniger einfach ist die Erfassung von Stückprozessen, bei denen durch Aufspaltung eines Werkstoffes mehrere Produktarten erzeugt werden. Da hierbei in einem Teilprozeß verschiedene Güterarten entstehen, liegt eine prozeßbedingte Divergenz vor. Tritt die Aufspaltung in mehrere Produktarten zwangsläufig auf, handelt es sich um einen $\mathrm{Kuppel-}$ proze $\left.\AA^{20}\right)$. Zur Abbildung dieses Vergenztyps im Input-Output-Ansatz kann eine der gemeinsam erzeugten Produktarten als Hauptprodukt definiert werden. Diesem Hauptprodukt werden alle Einsatzgüter des Kuppelprozesses zugerechnet $\left.{ }^{21}\right)$. Für die mit ihm anfallenden Nebenprodukte werden zusätzliche Güterzeilen und Prozeßspalten eingeführt. Die Spalten der Nebenprodukte enthalten nur Nullelemente, da für sie keine zusätzlichen Gütereinsätze erforderlich sind ${ }^{22}$ ). Dafür erscheinen in den Zeilen der Nebenprodukte jeweils in der Spalte des Hauptprodukts negative Einserelemente. Sie drücken aus, daß bei der Herstellung des Hauptprodukts nicht nur Güter eingesetzt, sondern andere Güter mit erzeugt werden. Ein "negativer" Einsatz bedeutet eine Güterentstehung ${ }^{23}$ ). Wenn z. B. durch die Aufspaltung des Rohstoffes $R_{1}$ ein Hauptprodukt $P_{2}$ und zwei Ne$170 \mathrm{f}$.

$\left.{ }^{19}\right) \mathrm{Zu}$ diesem Begriff vgl. Große-Oetringhaus (Fertigungstypologie)

$\left.{ }^{20}\right)$ Vgl. hierzu R i e b e 1 (Kuppelproduktion) $27 \mathrm{ff}$.

21) Diese Unterscheidung von Haupt- und Nebenprodukten begründet keine entsprechende Kostenverteilung.

22) Daran wird deutlich, daß für jedes Nebenprodukt ein „fiktiver" Teilprozeß definiert wird.

${ }^{23}$ ) Zur Bestimmung der Einsatz- und Ausbringungsmengen von Kuppelprozessen vgl. Abschnitt $4 \mathrm{c}$. 
benprodukte $P_{3}$ und $P_{4}$ erzeugt werden, kann der Materialfluß entsprechend Abbildung 4 wiedergegeben werden.
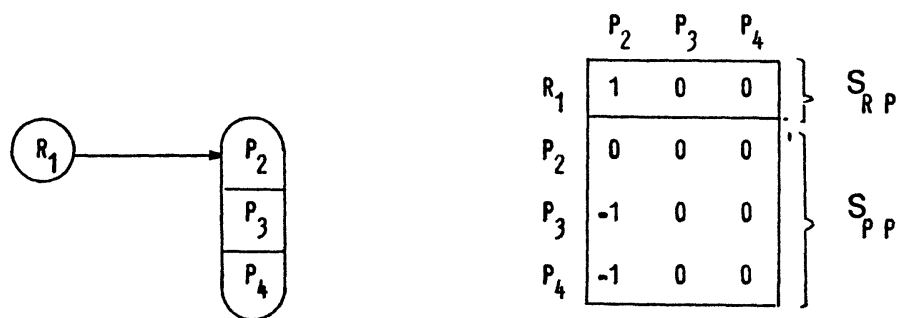

Abb. 4: Beispiel für Graph und Strukturmatrix eines prozeßbedingt divergierenden Stückprozesses.

Der Materialfluß wird als u mgruppierend bezeichnet, sofern in einem Teilprozeß mehrere Werkstoffarten eingesetzt und verschiedene Produktarten erzeugt werden. Hierbei treten Konvergenz und prozeßbedingte Divergenz nicht in nacheinander folgenden Teilprozessen, sondern in demselben Teilprozeß auf. Dies kommt in der aus Rohstoffeinsatzmatrix $S_{R P}$ und Produktverflechtungsmatrix $S_{P P}$ gebildeten Matrix darin zum Ausdruck, daß in der Spalte des Hauptprodukts mehrere positive und mindestens ein negatives Einserelement erscheinen. Die Spalten der Nebenprodukte enthalten lediglich Nullelemente (vgl. Abbildung 5).
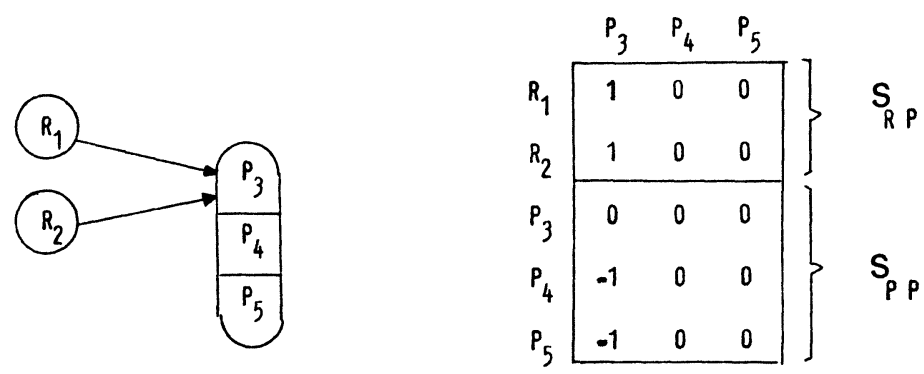

Abb. 5: Beispiel für Graph und Strukturmatrix eines umgruppierenden Stückprozesses.

Die Stückprozesse der Endproduktarten einer Unternehmung können sich aus mehreren Teilprozessen mit unterschiedlicher Struktur des Materialflusses zusammensetzen. Dann ergibt sich die Vergenz der gesamten Stückprozesse als Kombination der für die einzelnen Teilprozesse geltenden Vergenztypen. Mit Hilfe der gekennzeichneten Merkmale kann der Materialfluß für jeden Stückprozeß an der Rohstoffeinsatzmatrix $S_{R P}$ und der Produktverflechtungsmatrix $S_{P P}$ analysiert werden. 


\section{e) Organisationstypen der Fertigung}

Organisationstypen der Fertigung charakterisiert man durch die räumliche Anordnung der Produktiveinheiten (bzw. Maschinen) und die von der Arbeitsgangfolge der herzustellenden Endproduktarten abhängigen Transportbeziehungen zwischen den Produktiveinheiten ${ }^{24}$ ). Sie können im Input-Output-Ansatz durch die Arbeitseinsatzmatrix $S_{M P}$ wiedergegeben werden. Dabei sind einerseits die räumlich zu einer Werkstatt oder einer Fertigungsstraße zusammengefaßten Produktiveinheiten in unmittelbar aufeinanderfolgenden Zeilen aufzuführen und gegebenenfalls durch einen gemeinsamen Index zu kennzeichnen. Andererseits sind auch die zum Stückprozeß einer Endproduktart gehörenden Produktarten in unmittelbar aufeinanderfolgenden Spalten anzuordnen und gegebenenfalls mit einem gemeinsamen Index $\mathrm{zu}$ versehen. Diese Spaltenanordnung ist möglich, soweit in jedem Stückprozeß ein Vergenztyp vorherrscht und verschiedene Stückprozesse nicht durch divergierende und konvergierende Materialflüsse miteinander verbunden sind. Die zu einem Stückprozeß gehörenden (Zwischen- und End-)Produktarten führt man, soweit möglich, entsprechend ihrer Arbeitsgangfolge auf.

Aus der Anwendung des organisatorischen Verrichtungsprinzips oder des Objektprinzips ergeben sich als „reine“ Typen die Werkstatt- und die Fließfertigung ${ }^{25}$ ). Bei Werkstattfertigung sind die Maschinen, die gleichartige Verrichtungen ausführen, räumlich in Werkstätten zusammengefaßt. Die Produktarten durchlaufen die Werkstätten und gegebenenfalls mehrere Produktiveinheiten innerhalb einer Werkstatt in unterschiedlicher Reihenfolge. Dagegen weisen bei Fließfertigung alle auf einer Fertigungsstraße hergestellten Produkte und Produktarten dieselbe Maschinenfolge auf ${ }^{26}$ ). Die Produktiveinheiten sind in der Reihenfolge der Arbeitsgänge räumlich nacheinander angeordnet. Sofern jede $\mathrm{Zwischen-}$ oder Endproduktart nur in einer Produktiveinheit gefertigt werden kann, ist die Zuordnung der Produktarten zu den Produktiveinheiten, d. h. die Arbeitsverteilung, aus der Arbeitseinsatzmatrix $S_{M P}$ eindeutig erkennbar $^{27}$ ). Jede Zeile von $S_{M P}$ gibt an, welche verschiedenen Produktarten von derselben Produktiveinheit bearbeitet werden.

Bilden mehrere Produktiveinheiten $M_{1}, \ldots, M_{M}$ eine Fertigungsstraße,

24) Große-Oetring ha us (Fertigungstypologie) $269 \mathrm{ff}$.

${ }^{25}$ ) Zu diesen Typen vgl. Kos i ol (Aktionszentrum) $167 \mathrm{ff}$.; Gu t e n berg (Produktion) $96 \mathrm{ff}$.; S chä fer (Industriebetrieb) $171 \mathrm{ff}$.; Melle rowi cz (Industrie) $314 \mathrm{ff}$.; REFA (Arbeitsstudium 3) $174 \mathrm{ff}$.; $\mathrm{S} \mathrm{chweitzer}$ (Industriebetriebslehre) $118 \mathrm{ff}$; Große-Oetring ha u (Fertigungstypologie) $269 \mathrm{ff}$.

${ }^{26}$ ) Im Anschluß an $\mathrm{S}$ chäfer, Schweitzer, Große-Oetring$\mathrm{h}$ a u s u. a. wird der Begriff Fließfertigung als Oberbegriff für taktierte und untaktierte Fertigung nach dem Fließprinzip verwendet.

${ }^{27}$ ) Alternativen der Arbeitsverteilung lassen sich in $S_{M P}$ durch die Einführung von (binären) Zuordnungsvariablen wiedergeben, welche nur die Werte Null oder Eins annehmen können. 
auf der verschiedene Endproduktarten erzeugt werden, so lassen sich die Spalten für die zu einem Stückprozeß gehörenden (Zwischen- und End-)Produktarten $P_{i 1}, \ldots, P_{i S}$ in $S_{M P}$ nach der Maschinenfolge anordnen. Man erhält dann für jede auf der Fertigungsstraße erzeugte Endproduktart als Teilmatrix von $S_{M P}$ eine Einheitsmatrix (vgl. Abbildung 6).

\begin{tabular}{|c|c|c|c|c|c|c|c|c|c|c|c|c|c|c|}
\hline \multicolumn{4}{|c|}{ Endprodukt 1} & & \multicolumn{4}{|c|}{ Endprodukt 2} & \multicolumn{5}{|c|}{ Endprodukt 3} & \\
\hline$P_{11}$ & $P_{12}$ & $P_{13}$ & $P_{14}$ & & $P_{21}$ & $P_{22}$ & $P_{23}$ & $P_{24}$ & & 31 & $P_{32}$ & $P_{33}$ & $P_{34}$ & \\
\hline 1 & 0 & 0 & 0 & 1 & 1 & 0 & 0 & 0 & 1 & 1 & 0 & 0 & 0 & \\
\hline 12 & 1 & 0 & 0 & 1 & 0 & 1 & 0 & 0 & 0 & 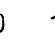 & 1 & 0 & 0 & $>$ \\
\hline 0 & 0 & 1 & 0 & 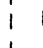 & 0 & 0 & 1 & 0 & 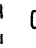 & 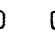 & 0 & 1 & 0 & \\
\hline 0 & 0 & 0 & 1 & & 0 & 0 & 0 & 1 & 10 & D & 0 & 0 & 1 & \\
\hline
\end{tabular}

Abb. 6: Beispiel für die Abbildung von Fließfertigung in der Arbeitseinsatzmatrix.

Diese Struktur der Arbeitseinsatzmatrix ergibt sich sowohl bei glatten als auch bei konvergierenden und divergierenden Stückprozessen, wenn mehrere Fertigungsstraßen zusammenlaufen oder sich eine Straße verzweigt.

Man faßt die Produktiveinheiten vor allem dann zu Werkstätten zusammen, wenn die Stückprozesse der Endproduktarten unterschiedliche Maschinenfolgen aufweisen. Gliedert man die Zeilen der Arbeitseinsatzmatrix $S_{\mathrm{MP}}$ nach Werkstätten und die Spalten nach den Stückprozessen der Endprodukte, so sind die Einserelemente in (fast) jeder Teilmatrix eines Stückprozesses anders angeordnet. Es lassen sich keine übereinstimmenden Merkmale dieser Teilmatrizen feststellen (vgl. Abbildung 7).

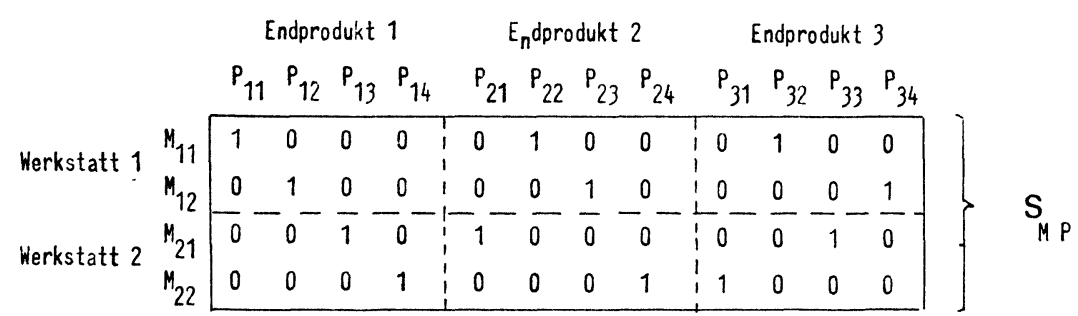

Abb. 7: Beispiel für die Abbildung von Werkstattfertigung in der Arbeitseinsatzmatrix.

Ein Zwischentyp liegt vor, wenn die Werkstätten bei allen Stückprozessen in gleicher Reihenfolge durchlaufen werden. Diesen Organisationstyp nennt man Werkstattfließfertigung. Bei ihm ist es möglich, die zu jedem Stückprozeß gehörigen Zwischen- und Endproduktarten entsprechend 
ihrer Bearbeitung in den Werkstätten zu gliedern. Dann wird durch die entsprechende Gliederung der Produktiveinheiten nach Werkstätten aus den sich für jeden Stückprozeß ergebenden Teilmatrizen ein übereinstimmendes Merkmal sichtbar. In jedem Stückprozeß treten lediglich in den Teilmatrizen von $S_{M P}$, die auf der jeweiligen Hauptdiagonalen liegen, positive Einserelemente auf. Abweichend von der Fließfertigung können jedoch die Einserelemente innerhalb dieser Teilmatrizen bei jedem Stückprozeß anders angeordnet sein. Hierin kommt die unterschiedliche Maschinenfolge der Stückprozesse bei gleichartiger Werkstattfolge zum Ausdruck (vgl. Abbildung 8).

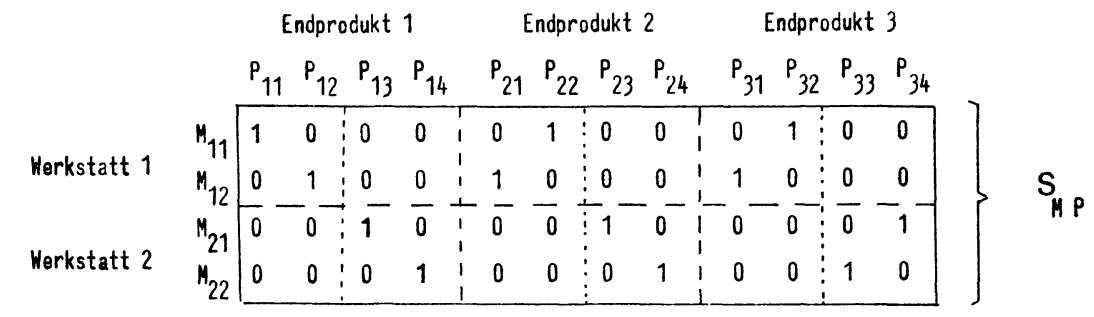

Abb. 8: Beispiel für die Abbildung von Werkstattfließfertigung in der Arbeitseinsatzmatrix.

Durch die Kombination von Fließ-, Werkstatt- und Werkstattfließfertigung für verschiedene Stückprozesse oder einzelne Teile von Stückprozessen lassen sich weitere gemischte Organisationstypen der Fertigung bilden.

Die bisherige Untersuchung macht deutlich, daß sich wichtige Typen der Produktionsstruktur auf der Grundlage des Input-Output-Ansatzes erfassen lassen. Abbildung 9 vermittelt einen Überblick über die Kennzeichen dieser Typen anhand der zerlegten Strukturmatrix $S^{*}$.

\section{Transformationsfunktionen derTeilprozesse}

Aus der Strukturmatrix S ergibt sich, zwischen welchen Güter- und Prozeßarten direkte Input-Output-Beziehungen bestehen. Die in einer Spalte von $S$ enthaltenen positiven Einserelemente geben an, welche Güterarten der Zeilen zur Herstellung der durch die Spalte bezeichnete Güterart eingesetzt werden. Um die quantitativen Beziehungen zwischen Einsatz und Ausbringung zu erfassen, ist die Strukturmatrix S durch Einsetzung der Transformationsfunktionen in die Direktverbrauchsmatrix F zu überführen. Hypothesen über die Existenz von Input-Output-Beziehungen in der Strukturmatrix werden durch Hypothesen über die Art dieser Beziehungen ersetzt. Zahl und Präzisionsgrad der erforderlichen Transformationsfunktionen werden vom Grad der Differenzierung zwischen Prozeßarten bestimmt. 


\begin{tabular}{|c|c|c|c|}
\hline Merknal der & Abbildung in & \multicolumn{2}{|c|}{ Ausprägungen der Strukturaatrix: } \\
\hline $\begin{array}{l}\text { Auftreten von } \\
\text { Produktionszyklen }\end{array}$ & $S$ & $\begin{array}{l}\text { - nichtzyklisch: } \\
\text { - zyklisch: }\end{array}$ & $\begin{array}{l}\text { lä日t sich zu Dreiecksnatrix unformen } \\
\text { läbt sich nicht zu Oreiecksmatrix unformen }\end{array}$ \\
\hline $\begin{array}{l}\text { Mehrstufigkeit } \\
\text { der Stückprozesse }\end{array}$ & $S_{P P}$ & $\begin{array}{l}\text { - oinstufig: } S_{P} \\
\text { - nebrstufig: } S_{P}\end{array}$ & $\begin{array}{l}=0 \\
\& 0\end{array}$ \\
\hline $\begin{array}{l}\text { Vergenz der } \\
\text { Stückprozesse }\end{array}$ & {$\left[\begin{array}{ll}\dot{S}_{R} & P \\
S_{P} & P\end{array}\right]$} & $\begin{array}{l}\text { - glatt: } \\
\text { - koavergierend: } \\
\text { - progranabedingt } \\
\text { divergierend: } \\
\text { - proze日bedingt } \\
\text { divergierend: } \\
\text { - ungruppierend: }\end{array}$ & 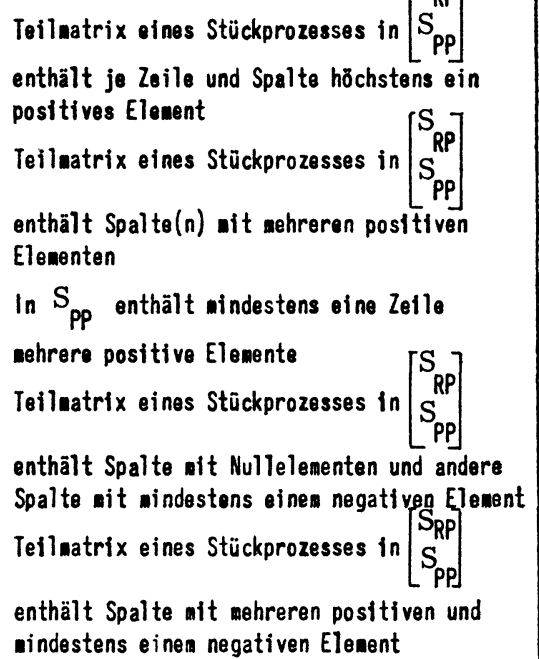 \\
\hline $\begin{array}{l}\text { Organi sationstyp } \\
\text { der Fertigung }\end{array}$ & $S_{M P}$ & $\begin{array}{l}\text { - FlieBfertigung: } \\
\text { - Werkstatt- } \\
\text { fertigung: } \\
\text { - WerkstattflieB- } \\
\text { fertigung: }\end{array}$ & $\begin{array}{l}\text { Für die auf einer Fertigungsstrabe hergestell- } \\
\text { ten Produkte erhält man Einheitsmatrizen } \\
\text { als Teilmatrizen von } \mathrm{S}_{M P} \\
\text { Die Teilmatrizen der Stückprozesse in } \mathrm{S}_{M P} \\
\text { weisen keine Übereinstimmung auf } \\
\text { Bei Untergliederung von } \mathrm{S}_{M P} \text { nach Werkstätten } \\
\text { und Stückprozessen sind lediglich die Teil- } \\
\text { aatrizen eines jeden Stückprozesses auf dessen } \\
\text { Hauptdiagonalen keine Nulluatrizen }\end{array}$ \\
\hline
\end{tabular}

Abb. 9: Uberblick über die Abbildung von Strukturmerkmalen des Produktionsprozesses in der Strukturmatrix. 


\section{a) Einvariablige Transformationsfunktionen}

Ein wichtiges Merkmal zur Unterscheidung mehrerer Klassen von Transformationsfunktionen kann in der Zahl ihrer unabhängigen Variablen gesehen werden. Bei einvariabligen Transformationsfunktionen hängt die Einsatzmenge $r_{i j}$ lediglich von der Ausbringungsmenge $r_{j}$ des $j$-ten Teilprozesses ab. Allgemein besitzen sie die Struktur:

$$
r_{i j}=g_{i j}\left(r_{j}\right)=f_{i j}\left(r_{j}\right) \cdot r_{j}
$$

Sofern eine proportionale Beziehung zwischen Einsatz- und Ausbringungsmenge besteht, handelt es sich um L e on tief-Funktionen :

$$
r_{i j}=a_{i j} \cdot r_{j} \quad a_{i j}=\text { konstant }
$$

Derartige Transformationsfunktionen werden insbesondere für den Einsatz an Werkstoffen behauptet ${ }^{28}$ ). Sie werden z.T. auch für den Einsatz an maschineller und menschlicher Arbeit verwendet. Dabei wird die Einsatzmenge an Arbeit $m$ (vereinfacht) durch die Arbeitszeit $t_{m j}$ im j-ten Teilprozeß gemessen und eine konstante oder durchschnittliche Arbeitsintensität unterstellt.

b) Mehrvariablige Transformationsfunktionen

Bestimmen neben der Ausbringungsmenge des Teilprozesses weitere Einflußgrößen die erforderliche Einsatzgütermenge, so ist die Transformationsfunktion mehrvariablig oder mehrdimensional ${ }^{29}$ ). Nach der Interdependenz der Bestimmungsgrößen für die Einsatzgütermenge lassen sich Transformationsfunktionen mit gegenseitig unabhängigen und gegenseitig abhängigen Einflußgrößen unterscheiden. Wenn die Einflüsse der verschiedenen Variablen auf die Höhe der Einsatzmenge voneinander unabhängig sind, setzt sich die Transformationsfunktion additiv aus mehreren Gliedern zusammen. Jedes Glied bezieht sich auf eine Variable. Zusätzlich kann ein Absolutglied auftreten ${ }^{30}$ ). Unterstellt man lineare Beziehungen zur Einsatzmenge, so erhält man Transformationsfunktionen der Art:

$r_{i j}=a_{i j} \cdot r_{j}+b_{i j}{ }^{1} \cdot e_{j}{ }^{1}+\ldots+b_{i j}{ }^{z} \cdot e_{j}{ }^{z}+c_{i j} \quad a_{i j}, b_{i j}{ }^{1}, \ldots, b_{i j}{ }^{z}, c_{i j}=$ konstant

Hierbei geben $r_{j}$ die Ausbringungsmenge und $e_{j}{ }^{1}, \ldots, e_{j}^{z}$ weitere Einflußgrößen des $\mathrm{j}$-ten Teilprozesses an. Als wichtige Einflußgrößen neben der Ausbringungsmenge können u. a. Betriebs- sowie Kalenderzeiten, Zahl der Lose, Schichtzahlen und Außentemperaturen auftreten ${ }^{31}$ ).

Dieser Typ von Transformationsfunktionen wird insbesondere in der

${ }^{28}$ ) $\mathrm{G}$ u t e n b e r g (Produktion) 337; H e in e n (Kostenlehre) $259 \mathrm{f}$.

29) Vgl. S c h we i t z e r (Kostenremanenz) 971 ff.

30) L a $B \mathrm{~m}$ a n n (Erlösrechnung) $85 \mathrm{ff}$.

31) L a $B$ mann (Erlösrechnung) $78 \mathrm{ff}$; F F a nke (Betriebsmodelle) $38 \mathrm{ff}$; $\mathrm{S}$ t effen (Elementarfaktoren) $129 \mathrm{ff}$; $\mathrm{Zschocke}$ (Betriebsökonometrie) 68. 
Einflußgrößenrechnung ${ }^{32}$ ) sowie in den Modellen von $\mathrm{Pich}$ le $\mathrm{r}$ und den hierauf basierenden Ansätzen ${ }^{33}$ ) zugrunde gelegt. Dabei ist es gelungen, in der Stahlindustrie und der chemischen Industrie Produktionsfunktionen mit einem hohen Grad an empirischer Bestätigung zu formulieren. Ein wichtiger Vorzug dieses Ansatzes liegt in der Anwendbarkeit der multiplen Regressionsrechnung. Ferner erleichtert die Linearität der Funktionen die Herleitung der Produktionsfunktion. Darüber hinaus stellen diese Transformationsfunktionen eine zweckmäßige Grundlage für die Kostenrechnung dar, weil jeder Bestimmungsgröße ein Anteil der Einsatzmenge und damit auch der Kosten zugerechnet werden kann $^{34}$ ).

In der Realität gibt es aber Prozesse, bei denen sich der Einfluß verschiedener Größen auf die Einsatzgütermenge nicht in voneinander unabhängige Glieder aufspalten läßt. Bei ihnen muß vielmehr von einer nichtadditiven Verknüpfung der Einflußgrößen in den Transformationsfunktionen ausgegangen werden. Eine derartige Interdependenz der Einflußgrößen besteht bei substituierbaren Einsatzgütern sowie bei einer Reihe von Verbrauchsfunktionen für den potentialgüterabhängigen Einsatz an Betriebsstoffen. Wenn bei der Erzeugung des j-ten Gutes $i=1, \ldots, n$ Einsatzgüter gegenseitig substituierbar sind, läßt sich die Transformationsfunktion allgemein durch ein System von Gleichungen der Art

$$
r_{i j}=g_{i j},\left(r_{j}, r_{1 j}, \ldots, r_{n j}\right)=f_{i j}\left(1, \frac{r_{1 j}}{r_{j}}, \ldots, \frac{r_{n j}}{r_{j}}\right) \cdot r_{j}
$$

wiedergeben $^{35}$ ). Ertragsgesetzliche, Cobb-Douglas- und CES-Funktionen ${ }^{36}$ ) gehören $\mathrm{zu}$ dieser Klasse von Transformationsfunktionen. Beispielsweise entspricht einer Cobb-Douglas-Funktion der Art

$$
\mathrm{r}_{\mathrm{j}}=\mathrm{a} \cdot \mathrm{r}^{\alpha}{ }_{1 \mathrm{j}} \cdot \mathrm{r} \beta_{2 \mathrm{j}}
$$

das System der beiden Gleichungen:

$$
r_{1 j}=\frac{r_{j}^{\frac{1}{\alpha}}-1}{\left(a \cdot r \beta_{2 j}\right)^{\frac{1}{\alpha}}} \cdot r_{j} \quad r_{2 j}=\frac{r_{j}^{\frac{1}{\beta}}-1}{\left(a \cdot r^{\alpha}{ }^{\frac{1}{j}}\right)^{\frac{1}{\beta}}} \cdot r_{j}
$$

32) Steffen/Stein ecke (Einflußgrößenrechnung) $155 \mathrm{ff}$;; Wart m a n n (Erfassung) 1414 ff., L a $\beta \mathrm{m}$ a n n (Erlösrechnung) $72 \mathrm{ff} . ; \quad F$ ra nke (Betriebsmodelle) $30 \mathrm{ff}$; $\mathrm{S}$ t e f f e n (Elementarfaktoren) $128 \mathrm{ff}$.

33) Vgl. insb. Pichler (Anwendung) $119 \mathrm{ff}$.; P i chle r (Matrizenrechnung) $157 \mathrm{ff}$; $\mathrm{P} \mathrm{i} \mathrm{chle} \mathrm{r} \mathrm{(Betriebskostenüberwachung)} 105 \mathrm{ff}$.; L a u e n s t e in (Probleme); K n o p (Kostenmodellierung); Z s chocke (Betriebsökonometrie) $67 \mathrm{ff}$.

${ }^{34}$ ) L a B m a n n (Erlösrechnung) $137 \mathrm{ff}$; F r a n k e (Betriebsmodelle) $120 \mathrm{ff}$.

$\left.{ }^{35}\right) \mathrm{K} 1$ o o c k (Input-Output-Modelle) 134.

$\left.{ }^{36}\right) \mathrm{Cob}$-Douglas- und CES-Funktionen werden im allgemeinen als aggregierte gesamtwirtschaftliche Produktionsfunktionen verwendet. Jedoch ist nicht ausgeschlossen, daß mit ihnen auch Teilprozesse mit substituierbaren Einsatzgütern (z. B. in der chemischen Industrie) abgebildet werden können. 
In den Verbrauchsfunktionen von Gutenberg, Kilger und Heinen wird angenommen, daß die Einsatzmenge von Betriebsstoffen an Potentialgütern (wie Maschinen) von deren technischen Eigenschaften, Arbeitsintensität und Fertigungszeit abhängig ist. Bei Konstanz der technischen Eigenschaften wird die für eine Verrichtung des Potentialguts $\mathrm{m}$ einzusetzende Betriebsstoffmenge $\varrho_{\mathrm{im}}$ von der Intensität $\mathrm{d}_{\mathrm{m}}$ bestimmt ${ }^{37}$ ):

$$
\varrho_{\mathrm{im}}=\mathrm{f}_{\mathrm{im}}\left(\mathrm{d}_{\mathrm{m}}\right)
$$

Die Anzahl der Verrichtungen, die das Potentialgut $\mathrm{m}$ in einer Produktionsperiode durchführt, entspricht dem Produkt aus Intensität $\mathrm{d}_{\mathrm{m}}$ (= Zahl der Verrichtungen je Zeiteinheit) und Fertigungszeit $t_{m}$. Somit erhält man für den Verbrauch an Betriebsstoffen in der Produktionsperiode die Verbrauchsfunktion ${ }^{38}$ ):

$$
\mathrm{r}_{\mathrm{im}}=\mathrm{f}_{\mathrm{im}}\left(\mathrm{d}_{\mathrm{m}}\right) \cdot \mathrm{d}_{\mathrm{m}} \cdot \mathrm{t}_{\mathrm{m}}
$$

Die Beziehungen zwischen den Leistungsabgaben des Potentialguts $\mathrm{m}$, die durch seine Fertigungszeit $t_{m}$ gemessen werden ${ }^{39}$ ), und den auf ihm hergestellten Produktmengen $r_{j}$ werden nach Kilger ${ }^{40}$ ) durch eine weitere Transformationsfunktion abgebildet. Er geht davon aus, daß zur Herstellung einer Einheit der j-ten Zwischen- oder Endproduktart von dem m-ten Potentialgut $\alpha_{\mathrm{mj}}$ Verrichtungen durchgeführt werden müssen. Somit gilt für diese Art von Transformationsfunktionen:

$$
\mathrm{t}_{\mathrm{mj}}=\frac{1}{\mathrm{~d}_{\mathrm{m}}} \cdot \alpha_{\mathrm{mj}} \cdot \mathrm{r}_{\mathrm{j}}
$$

In Erweiterung dieses Ansatzes untersucht Heinen ${ }^{41}$ ) die Abhängigkeit der Einsatzmenge an Betriebsstoffen von den Intensitätsschwankungen der Potentialgüter. Als kleinsten Teilprozeß betrachtet er Elementarkombinationen, für die sich eindeutige Input-Output-Beziehungen bestimmen lassen. Die Zeitdauer $t_{j k}$ einer Elementarkombination $j$ auf der $k$-ten Produktionsstufe kann als Maß der (Durchschnitts-)Intensität des Potentialguts bzw. der Produktiveinheit interpretiert werden. Neben ihr werden als mögliche Einflußgrößen des potentialgüterabhängigen Gütereinsatzes die Ausbringungsmenge $\lambda_{\mathrm{jk}}$ bei einmaligem Vollzug der Elementarkombination sowie bei substitutionalen Prozessen die Intensitätsverläufe $\frac{\mathrm{db}_{\mathrm{jk}}^{(\mathrm{u})}}{\mathrm{dt}}$ eines (oder mehrerer) anderen Potentialguts u eingeführt, das an derselben Elementarkombination mitwirkt. Betrachtet man die Anzahl $\mathbf{w}_{\mathrm{jk}}$ der vollzogenen Elementarkombinationen als Maß für die Einsatzmenge des Poten-

\footnotetext{
${ }^{37}$ ) Gut e n berg (Produktion) $330 \mathrm{f}$.

${ }^{38}$ ) Vgl. Kilger (Produktionstheorie) $63 \mathrm{ff}$;; S chweitzer/Kü p per (Produktionstheorie) $87 \mathrm{ff}$

${ }^{39}$ ) Dies entspricht weitgehend dem Vorgehen der Praxis.

${ }^{40} \mathrm{~K}$ K $1 \mathrm{~g}$ e r (Produktionstheorie) 65.

41) $\mathrm{H}$ e in e n (Kostenlehre) $220 \mathrm{ff}$.
} 
tialguts in einer Periode, so erhält man als allgemeine Verbrauchsfunktion für den Einsatz an Betriebsstoffen ${ }^{42}$ ):

$$
r_{i j k}=f_{i j k}\left(t_{j k}, \lambda_{j k}, \frac{d b_{j k}^{(u)}}{d t}\right) \cdot w_{j k}
$$

Kardinale Hypothesen über die Art der Verknüpfung dieser Einflußgrößen formuliert Heinen nicht.

Ferner entwickelt Heinen Transformationsfunktionen für die Beziehungen $z$ wischen der Anzahl Wiederholungen $w_{j k}$ einer Elementarkombination $\mathrm{jk}$ und der $Z$ wischenproduktmenge $r_{\mathrm{k}}$. Er unterstellt, daß für die Wiederholungszahl $w_{j k}$ neben der $Z$ wischenproduktmenge $r_{k}$ ein Ausschußkoeffizient $c_{j k}$, die Ausbringungsmenge $\lambda_{j k}$ je einmaligem Vollzug der Elementarkombination sowie ein Koeffizient $v_{j k}$ der Arbeitsverteilung bestimmend sind:

$$
w_{j k}=\frac{c_{j k}}{\lambda_{j k}} \cdot v_{j k} \cdot r_{k}
$$

Die wichtigsten Klassen von Transformationsfunktionen sind in Abbildung 10 zusammenfassend wiedergegeben.

4. Bestimmung der Produktionsfunktion der Unterne h m ung

a) Herleitung der Produktionsfunktion auf der Basis von Leontief- und von Verbrauchsfunktionen

Strukturmatrix und Transformationsfunktionen bilden die Grundlage für die Herleitung der Produktionsfunktion einer Unternehmung. In einem vereinfachten Beispiel kann man unterstellen, daß der Einsatz an Betriebsstoffen (B) lediglich von den Leistungsabgaben der Produktiveinheiten (M) direkt abhängig ist. Die Leistungsabgaben der Produktiveinheiten (bzw. der in ihnen eingesetzten Potentialgüter) sowie die Einsatzmengen an Rohstoffen $(R)$ und (Zwischen-)Produkten $(P)$ hängen nur von den in der Unternehmung hergestellten Produktmengen $(\mathrm{P})$ direkt ab. Unter diesen Annahmen sind in der zerlegten Strukturmatrix $S^{*}$ (5) lediglich die Teilmatrizen $\mathrm{S}_{\mathrm{BM}}, \mathrm{S}_{\mathrm{RP}}, \mathrm{S}_{\mathrm{MP}}$, und $\mathrm{S}_{\mathrm{PP}}$ keine Nullmatrizen:

$$
S^{*}=\left[\begin{array}{llll}
0_{R R} & 0_{R B} & 0_{R M} & s_{R P} \\
0_{B R} & 0_{B B} & s_{B H} & 0_{B P} \\
0_{M R} & 0_{A B} & 0_{M H} & s_{M P} \\
0_{P R} & 0_{P B} & 0_{P H} & s_{P P}
\end{array}\right]
$$

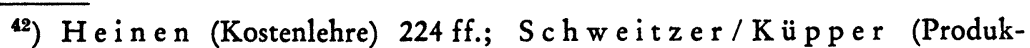
tionstheorie) $113 \mathrm{ff}$. 


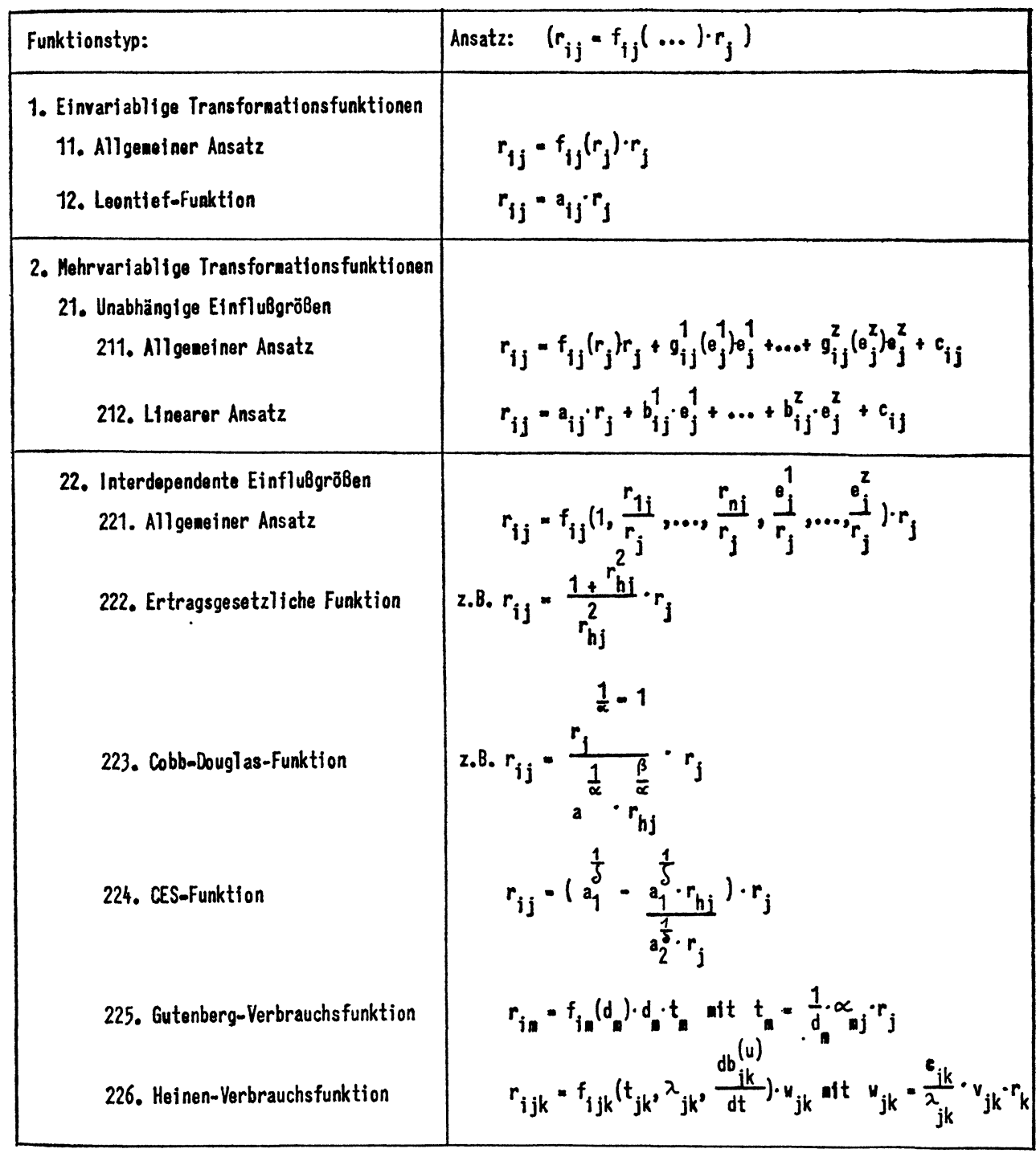

Abb. 10: Überblick über wichtige Klassen von Transformationsfunktionen. 
Aufgrund des Input-Output-Ansatzes (4) gelangt man zu folgendem allgemeinen Produktionsmodell:

$\left[\begin{array}{l}r_{R} \\ r_{B} \\ r_{M} \\ r_{P}\end{array}\right]=\left[\begin{array}{cccc}E_{R R} & o_{R B} & 0_{R M} & -F_{R P} \\ O_{B R} & E_{B B} & -F_{B M} & 0_{B P} \\ O_{M R} & 0_{M B} & E_{M M} & -F_{M P} \\ O_{P R} & 0_{P B} & 0_{P M} & \left(E_{P P}-F_{P P}\right)\end{array}\right]^{-1} \cdot\left[\begin{array}{c}x_{R}+\Delta 1_{R} \\ x_{B}+\Delta 1_{B} \\ 0 \\ x_{P}+\Delta 1_{P}\end{array}\right]$

In ihm geben die einzelnen Vektoren $r, x$ und $\Delta 1$ die Einsatz- bzw. Ausbringungsmengen, die Absatzmengen und die Lagerbestandsänderungen der Güterarten wieder. Die Teilmatrizen von $S^{*}$ bilden die Existenz von Direktverbrauchsmatrizen für die Beziehungen zwischen den Prozeßarten ab. Zusätzlich wird angenommen, daß lediglich Roh- und Betriebsstoffe sowie Zwischen- und Endprodukte abgesetzt und gelagert werden können. Dann erhält man nach den Regeln für die Inversion zerlegter Matrizen ${ }^{43}$ ) aus (10) folgendes Gleichungssystem ${ }^{44}$ ):

$\left[\begin{array}{l}r_{R} \\ r_{B} \\ r_{M} \\ r_{P}\end{array}\right]=\left[\begin{array}{lllr}E_{R R} & 0_{R B} & 0_{R M} & F_{R P} \cdot\left(E_{P P}-F_{P P}\right)^{-1} \\ O_{B R} & E_{B B} & F_{B M} & F_{B M} \cdot F_{M P} \cdot\left(E_{P P}-F_{P P}\right)^{-1} \\ O_{M R} & 0_{M B} & E_{M M} & F_{M P} \cdot\left(E_{P P}-F_{P P}\right)^{-1} \\ O_{P R} & 0_{P B} & 0_{P M} & \left(E_{P P}-F_{P P}\right)^{-1}\end{array}\right] \cdot\left[\begin{array}{c}x_{R}+\Delta 1_{R} \\ x_{B}+\Delta 1_{B} \\ 0 \\ x_{P}+\Delta 1_{P}\end{array}\right]$

In diesem Gleichungssystem sind für die direkten Input-Output-Beziehungen der Teilprozesse bestimmte Arten von Transformationsfunktionen zugrunde zu legen. Die Abhängigkeit der Einsatzmengen an Rohstoffen und Zwischenprodukten von den Zwischen- oder Endproduktmengen kann durch Leontief-Transformationsfunktionen (6)

$$
r_{i j}=a_{i j} \cdot r_{j} \quad a_{i j}=\text { konstant }
$$

erfaßt werden. Als Transformationsfunktionen für den Verbrauch an Betriebsstoffen werden Verbrauchsfunktionen entsprechend (8) angenommen:

$$
r_{i m}=f_{i m}\left(d_{m}\right) \cdot d_{m} \cdot t_{m}
$$

Für die Beziehungen zwischen den Fertigungszeiten der Produktiveinheiten (bzw. Potentialgüter) und den hergestellten Produktmengen können Transformationsfunktionen der Art (9)

$$
t_{m j}=\frac{1}{d_{m}} \cdot \alpha_{m j} \cdot r_{j}
$$

${ }^{43}$ ) Vgl. z.B. Ayres (Matrices) 56 f.; Faddejew/Faddejewa (Algebra) $201 \mathrm{f}$.

44) Vgl. auch K l o o c k (Input-Output-Analyse) $1957 \mathrm{ff}$. 
unterstellt werden. Um zur Produktionsfunktion der Unternehmung zu gelangen, sind darüber hinaus bestimmte Ausprägungen der Rohstoffeinsatzmatrix $S_{R P}$, der Betriebsstoffeinsatzmatrix $S_{B M}$, der Arbeitseinsatzmatrix $S_{M P}$ und der Produktverflechtungsmatrix $S_{P P}$ vorzugeben. Als Beispiel wird die in Abbildung 11 dargestellte einfache Struktur eines konvergierenden Stückprozesses zugrunde gelegt.
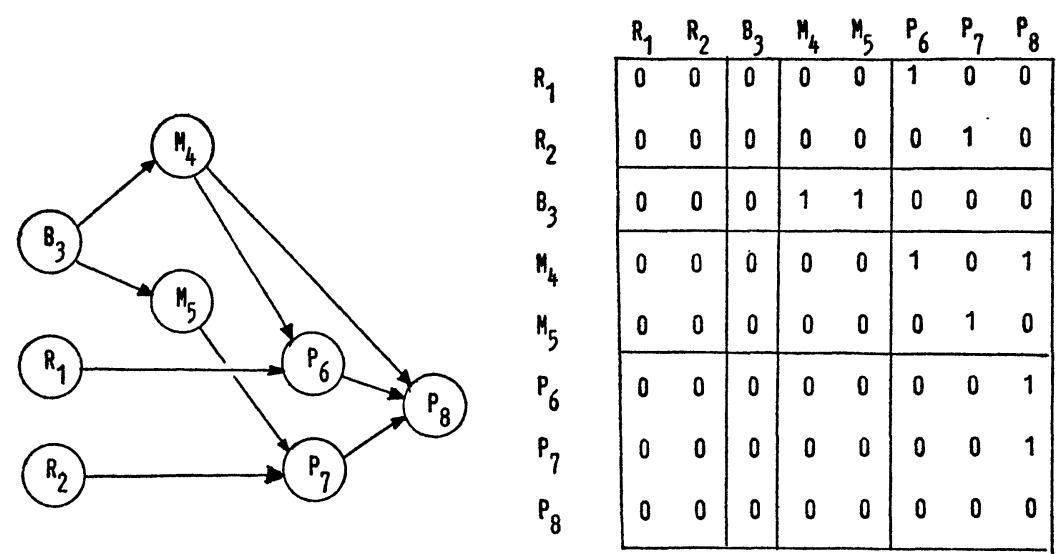

Abb. 11: Beispiel der Struktur eines konvergierenden Stückprozesses.

Aufgrund dieser Produktionsstruktur und der in (6), (8) und (9) angenommenen Arten von Transformationsfunktionen erhält man das InputOutput-Modell (12):

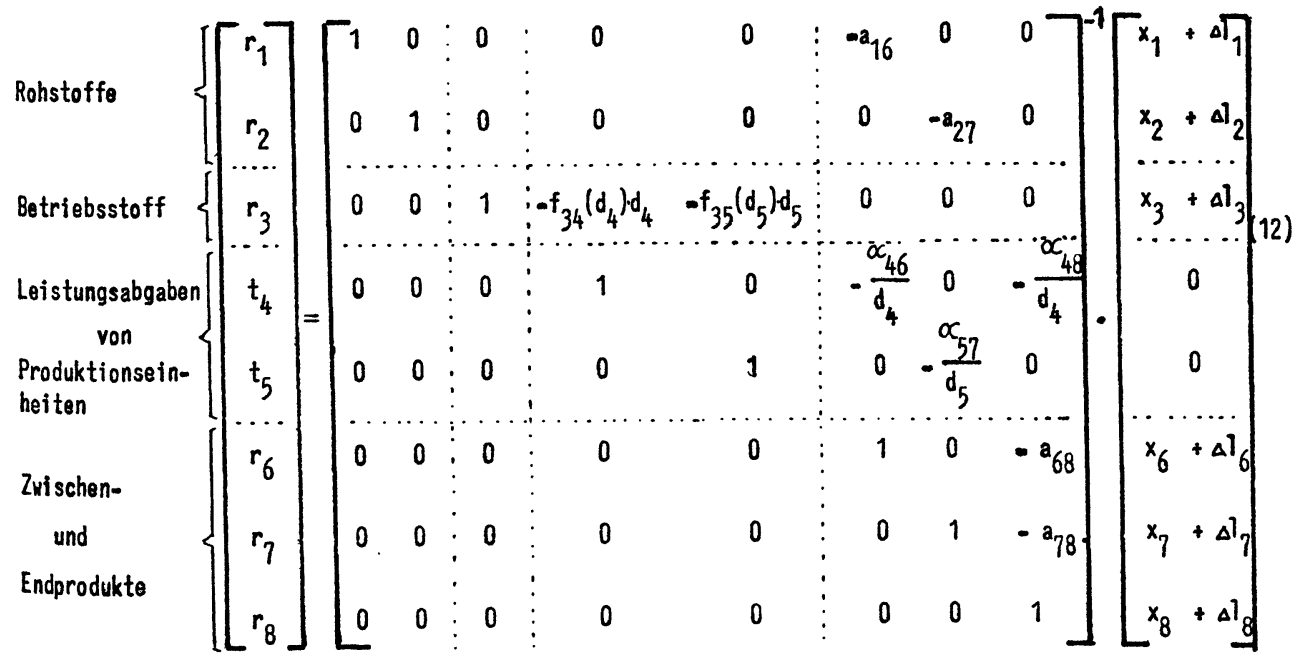


Hieraus ergibt sich entsprechend (11):

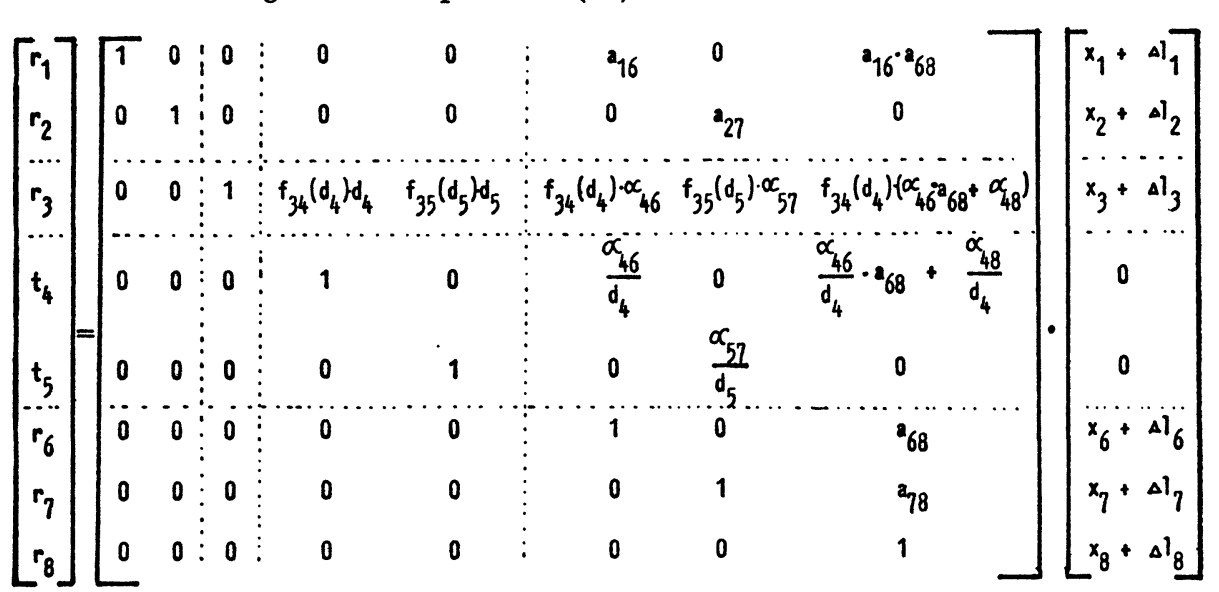

Die ersten fünf Gleichungen des Systems (13) geben die quantitativen Beziehungen zwischen dem originären Gütereinsatz und dem Ouput der Unternehmung wieder. Sie lassen sich daher als Produktionsfunktion der Unternehmung interpretieren. Bei der angenommenen nichtzyklischen Produktionsstruktur können Teilproduktionsfunktionen für die Leistungsabgaben der Produktiveinheiten sowie den Einsatz an Rohstoffen und Betriebsstoffen sukzessiv bestimmt werden ${ }^{45}$ ).

Die Teilproduktionsfunktion für den Betriebsstoffeinsatz aus

$r_{3}=x_{3}+\Delta l_{3}+f_{34}\left(d_{4}\right) \cdot \alpha_{46}\left(x_{6}+\Delta l_{6}\right)+f_{35}\left(d_{5}\right) \cdot \alpha_{57}\left(x_{7}+\Delta l_{7}\right)+f_{34}\left(d_{4}\right) \cdot\left(\alpha_{46} \cdot a_{68}+\alpha_{48}\right)\left(x_{8}+\Delta 1_{8}\right)$

ist nichtlinear, sofern die Intensitäten der Produktiveinheiten kontinuierlich verändert werden können. Sie läßt sich durch einen linearen Ansatz approximativ wiedergeben, wenn man bei jeder Produktiveinheit lediglich bestimmte Intensitätsgrade berücksichtigt $\left.{ }^{46}\right)$. Dann ist für jeden Intensitätsgrad einer Produktiveinheit ein eigener Teilprozeß mit konstanter Intensität zu definieren. Ferner sind die Zwischen- oder Endprodukte, die auf der betreffenden Produktiveinheit hergestellt werden, danach zu unterscheiden, mit welchem Intensitätsgrad sie gefertigt werden. Kann z. B. die Produktiveinheit $M_{5}$ mit den (konstanten) Intensitätsgraden $\bar{d}_{51}$ und $\overline{\mathrm{d}}_{52}$ eingesetzt werden, so gilt für den Betriebsstoffeinsatz in $\mathrm{M}_{5}$ die Beziehung:

$$
r_{35}=f_{3,51}\left(\partial_{51}\right) \partial_{51} t_{51}+f_{3,52}\left(\partial_{52}\right) t_{52} t_{52}=\beta_{51} \cdot t_{51}+\beta_{52} \cdot t_{52} \quad \beta_{51} \cdot \beta_{52}=\text { konstant }
$$

In den Teilprozessen $\mathrm{M}_{51}$ bzw. $\mathrm{M}_{52}$ werden die Zwischenproduktmen-

45) Vgl. Kl oock (Input-Output-Modelle) $126 \mathrm{ff}$; S chweitzer/ $\mathrm{K}$ ü p p e r (Produktionstheorie) $145 \mathrm{ff}$.

46) Vgl. J a c o b (Produktionsplanung) $249 \mathrm{ff}$. 
gen $r_{71}$ bzw. $r_{72}$ erzeugt. Beide Zwischenproduktarten sind identisch. Deshalb ist eine zusätzliche Gleichung einzuführen, welche die Verteilung der Zwischenproduktmenge $r_{7}$ auf $r_{71}$ und $r_{72}$ angibt. Wenn man $v_{j}$ als Verteilungskoeffizienten mit den Eigenschaften

$$
0 \leqq \mathrm{v}_{\mathbf{j} \delta} \leqq 1 \text { und } \sum_{\delta} \mathrm{v}_{\mathbf{j} \delta}=1
$$

definiert, der den Anteil der beim Intensitätsgrad $\delta$ erstellten Produktmenge $r_{j \delta}$ an $r_{j}$ angibt, lautet die Verteilungsgleichung in diesem Beispiel:

$$
r_{7}=v_{71} \cdot r_{71}+v_{72} \cdot r_{72}
$$

Unter Verwendung der Teilprozesse für mehrere Intensitätsgrade je Produktiveinheit und Einfügung der entsprechenden Verteilungsgleichungen für die Produkte erhält man einen linearen Input-Output-Ansatz.

b) Berücksichtigung mehrvariabliger Transformationsfunktionen mit gegenseitig unabhängigen Einflußgrößen

Umfaßt die Direktverbrauchsmatrix $F$ mehrvariablige Transformationsfunktionen mit interdependenten Variablen, so können bei Bestimmung der Gesamtverbrauchsmatrix $(\mathrm{E}-\mathrm{F})^{-1}$ schwierige Kalkülprobleme auftreten ${ }^{47}$ ). Vielfach lassen sich jedoch nichtlineare Transformationsfunktionen in der beschriebenen Weise durch mehrere Teilprozesse mit linearen Transformationsfunktionen approximativ ersetzen. So kann ein Prozeß mit kontinuierlich substituierbaren Einsatzgütern in mehrere Teilprozesse mit verschiedenen, jeweils konstanten Mengenrelationen der Einsatzgüter zerlegt werden.

Bei mehrvariabligen Transformationsfunktionen mit gegenseitig unabhängigen Einflußgrößen erscheint ein anderes Vorgehen zweckmäßig. Im Fall linearer Transformationsfunktionen der Art (7)

$$
r_{i j}=a_{i j} \cdot r_{j}+b_{i j}{ }^{1} \cdot e_{j}{ }^{1}+\ldots+b_{i j}{ }^{z} \cdot e_{j}^{z}+c_{i j}
$$

in denen neben den Ausbringungsmengen $r_{j}$ unabhängige Einflußgrößen $e_{j}{ }^{1}$, $\ldots, e_{j}{ }^{z}$ und Absolutglieder $c_{i j}$ auftreten, ist das Input-Output-Gleichungssystem wie folgt zu erweitern:

$$
\begin{aligned}
& {\left[\begin{array}{c}
r_{1} \\
\vdots \\
r_{n}
\end{array}\right]=\left[\begin{array}{lll}
a_{11} & \cdots & a_{1 n} \\
\vdots & & \vdots \\
a_{n 1} & \cdots & a_{n n}
\end{array}\right] \cdot\left[\begin{array}{c}
r_{1} \\
\vdots \\
r_{n}
\end{array}\right]+\left[\begin{array}{lll}
b_{11} & \cdots & b_{1 n}{ }^{1} \\
\vdots & & \vdots \\
b_{n 1} & \cdots & b_{n n}
\end{array}\right] \cdot\left[\begin{array}{c}
e_{1}{ }^{1} \\
\vdots \\
e_{n}
\end{array}\right]+\cdots+\left[\begin{array}{lll}
b_{11}{ }^{2} & \cdots & b_{1 n}{ }^{2} \\
\vdots & & \vdots \\
b_{n 1}{ }^{2} & \cdots & b_{n n}{ }^{2}
\end{array}\right] \cdot\left[\begin{array}{l}
e_{1}{ }^{2} \\
\vdots \\
e_{n}{ }^{2}
\end{array}\right]} \\
& +\left[\begin{array}{ccc}
c_{11} & \cdots & c_{1 n} \\
\vdots & & \vdots \\
c_{n 1} & \cdots & c_{n n}
\end{array}\right] \cdot\left[\begin{array}{l}
1 \\
1 \\
\vdots \\
1
\end{array}\right]+\left[\begin{array}{c}
x_{1} \\
\vdots \\
x_{n}
\end{array}\right]+\left[\begin{array}{l}
\Delta l_{1} \\
\vdots \\
\Delta l_{n}
\end{array}\right]
\end{aligned}
$$

${ }^{47}$ ) K 1 o o ck (Produktionstheorie) 64. 
Hierdurch wird es möglich, Einflußgrößen im Input-Output-Ansatz zu berücksichtigen, die nicht als Ausbringungsmengen von Teilprozessen interpretierbar sind.

\section{c) Erfassung von Kuppelprozessen}

Die Erfassung von Kuppelprozessen kann durch eine Erweiterung des Beispiels von Abbildung 11 beschrieben werden. Es wird angenommen, daß Produkt $P_{6}$ in einem Kuppelprozeß erzeugt wird, in dem auch das Produkt $\mathrm{P}_{6} *$ anfällt. Letzteres kann zum Endprodukt $\mathrm{P}_{9}$ weiterverarbeitet werden und wird als Nebenprodukt des Kuppelprozesses definiert. Zwischen den Ausbringungsmengen der beiden Kuppelprodukte bestehe das $s$ t a r r e Mengenverhältnis:

$$
\mathrm{r}_{6}^{*}=\mathrm{k}_{6} \cdot \mathrm{r}_{6} \quad \mathrm{k}_{6}=\text { konstant }
$$

Kuppelprozesse mit veränderlichem Mengenverhältnis können in mehrere Prozesse mit jeweils starrem Mengenverhältnis und in der Regel unterschiedlichen Einsatz- und Verfahrensbedingungen zerlegt werden ${ }^{48}$ ).

$\mathrm{Da}$ eine nichtzyklische Produktionsstruktur unterstellt wird, braucht die Erweiterung des Input-Output-Ansatzes lediglich an den Teilmatrizen $F_{P P}$ und $\left(E_{\mathrm{PP}}-\mathrm{F}_{\mathrm{PP}}\right)^{-1}$ gekennzeichnet $\mathrm{zu}$ werden. Abbildung 12 stellt die Verflechtungen unter den Produkten des erweiterten Beispiels und die hieraus folgende Produktverflechtungsmatrix $S_{P P}$ dar.
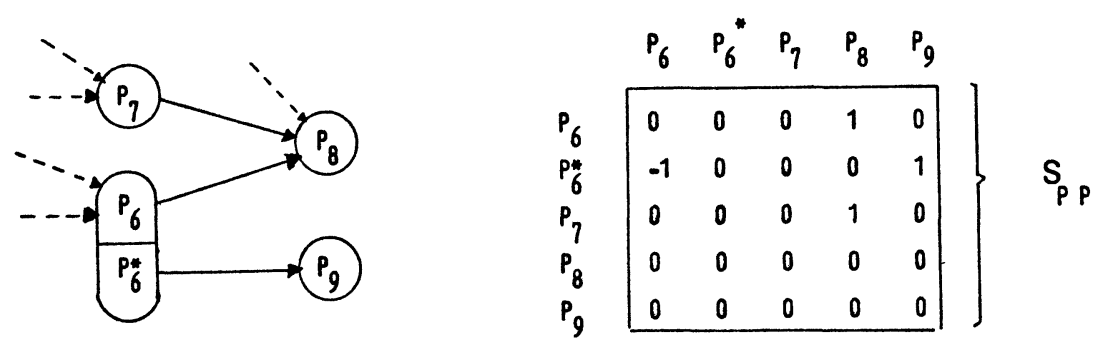

Abb. 12: Graph und Produktverflechtungsmatrix für Beispiel eines Kuppelprozesses.

In das Input-Output-Modell ist für das Nebenprodukt $\mathrm{P}_{6}^{*}$ eine zusätzliche Spalte einzuführen, die lediglich Nullen enthält. Ferner ist eine weitere Gleichung zu formulieren. Diese gibt an, in welchem Unfang die Ausbringungsmenge $r_{0}^{*}$ des Nebenprodukts wiedereingesetzt, abgesetzt oder auf Lager gelegt wird. Anstelle von $r_{6}^{*}$ setzt man jedoch in dieser Gleichung $k_{6} \cdot r_{6}$ aus (14) ein. Für das behandelte Beispiel gilt demnach:

$$
0=-\mathrm{k}_{6} \cdot \mathrm{r}_{6}+\mathrm{a}_{60}^{*} \cdot \mathrm{r}_{9}+\mathrm{x}_{6}{ }^{*}+\Delta \mathrm{l}_{6}{ }^{*}
$$

${ }^{48}$ ) R i e b e 1 (Kuppelproduktion) $91 \mathrm{ff}$. 
Charakteristisch ist hierbei, daß im Vektor $\mathrm{r}_{P}$ an die Stelle der Variablen $\mathrm{r}_{\mathrm{a}}{ }^{*}$ eine Null gesetzt wird. Dem entspricht, daß die Ausbringungsmenge $r_{6}{ }^{*}$ keine Variable darstellt, sondern durch die Ausbringungsmenge des Hauptprodukts eindeutig bestimmt ist. Fügt man diese Gleichung in den Input-Output-Ansatz ein, so erhält man für die Zwischen- und Endprodukte folgendes Gleichungssystem:

$$
\left[\begin{array}{c}
r_{6} \\
0 \\
r_{7} \\
r_{8} \\
r_{9}
\end{array}\right]=\left[\begin{array}{ccccc}
0 & 0 & 0 & a_{68} & 0 \\
-k_{6} & 0 & 0 & 0 & a_{69}^{*} \\
0 & 0 & 0 & a_{78} & 0 \\
0 & 0 & 0 & 0 & 0 \\
0 & 0 & 0 & 0 & 0
\end{array}\right] \cdot\left[\begin{array}{c}
r_{6} \\
0 \\
r_{7} \\
r_{8} \\
r_{9}
\end{array}\right]+\left[\begin{array}{cc}
x_{6}+\Delta l_{6} \\
x_{6}^{*}+\Delta l_{6}^{*} \\
x_{7}+\Delta l_{7} \\
x_{8}+\Delta l_{8} \\
x_{9}+\Delta l_{9}
\end{array}\right]
$$

Dieses Gleichungssystem wird nach dem Vektor $\mathrm{r}_{\mathrm{P}}$ aufgelöst:

$$
\left[\begin{array}{c}
r_{6} \\
0 \\
r_{7} \\
r_{8} \\
r_{9}
\end{array}\right]=\left[\begin{array}{ccccc}
1 & 0 & 0 & -a_{68} & 0 \\
k_{6} & 1 & 0 & 0 & -a_{6}^{*} \\
0 & 0 & 1 & -a_{78} & 0 \\
0 & 0 & 0 & 1 & 0 \\
0 & 0 & 0 & 0 & 1
\end{array}\right]^{-1}\left[\begin{array}{c}
x_{6}+\Delta 1_{6} \\
x_{6}^{*}+\Delta i_{6}^{*} \\
x_{7}+\Delta l_{7} \\
x_{8}+\Delta 1_{8} \\
x_{9}+\Delta l_{9}
\end{array}\right]=\left[\begin{array}{ccccc}
1 & 0 & 0 & a_{68} & 0 \\
-k_{6} & 1 & 0 & -k_{6} a_{68} & a_{69}^{*} \\
0 & 0 & 1 & a_{78} & 0 \\
0 & 0 & 0 & 1 & 0 \\
0 & 0 & 0 & 0 & 1
\end{array}\right]\left[\begin{array}{c}
x_{6}+\Delta l_{6} \\
x_{6}^{*}+\Delta l_{6}^{*} \\
x_{7}+\Delta l_{7} \\
x_{8}+\Delta l_{8} \\
x_{9}+\Delta l_{9}
\end{array}\right]
$$

Bei diesem Ansatz ist aufgrund der starren Mengenrelation zwischen $r_{6}$ und $r_{6}{ }^{*}$ die Zahl der Gleichungen größer als die Zahl der Ausbringungsmengenvariablen. Ferner enthält die Gesamtverbrauchsmatrix ( $E_{P P}-$ $\left.\mathrm{F}_{\mathrm{PP}}\right)^{-1}$ negative Elemente. Daraus folgt, daß nicht alle Werte der Absatzund Lagerbestandsänderungsvariablen beliebig wählbar sind. Neben den Ausbringungsmengen ist eine Absatz- oder Lagerbestandsänderungsvariable des Kuppelprozesses als abhängige Variable zu behandeln. In der Regel wird man die Lagerbestandsänderung des Nebenprodukts als weitere abhängige Variable wählen. Ferner sind die Absatz- und Lagerbestandsänderungsvariablen der Kuppelprodukte in ihrem Wertebereich begrenzt, sofern nur Lagerbestandserhöhungen bzw. der Abbau eines gegebenen Lagerbestands zulässig sind. Wählt man im betrachteten Beispiel $\Delta \mathrm{l}_{6}^{* *}$ als weitere abhängige Variable, und kann ein Lagerbestand $\mathrm{L}_{6}$ * abgebaut werden, so folgt aus der Gleichung für das Nebenprodukt in (15) die Ungleichung:

$$
\left(\Delta 1_{6}^{*}=\right) k_{6} \cdot\left(x_{6}+\Delta 1_{6}\right)-x_{6}{ }^{*}+k_{6} \cdot a_{68} \cdot\left(x_{8}+\Delta l_{8}\right)-a_{69}^{*} \cdot\left(x_{8}+\Delta l_{9}\right) \geqq-L_{6}{ }^{*}
$$

Ungleichung (16) grenzt den zulässigen Wertebereich der unabhängigen Variablen ein. 


\section{Zusammenf assung}

Die Untersuchung zeigt, daß der Input-Output-Ansatz eine fruchtbare Grundlage für eine realitätsnahe Abbildung des Produktionsprozesses der Unternehmung darstellt. Seine wesentlichen Komponenten bestehen in der Strukturmatrix und den Transformationsfunktionen. Die Strukturmatrix bildet die Existenz direkter Beziehungen zwischen den einzelnen Güterarten und Teilprozessen ab. In ihr können wichtige Strukturmerkmale industrieller Fertigungsprozesse wiedergegeben werden. So kann die Strukturmatrix bei zyklischen Prozessen nicht auf die Form einer Dreiecksmatrix gebracht werden. Mehrstufige Prozesse sind daran erkennbar, daß die Produktverflechtungsmatrix keine Nullmatrix darstellt. Die Vergenz der Stückprozesse läßt sich durch die Besetzung der Zeilen und Spalten in der Rohstoffeinsatzmatrix und der Produktverflechtungsmatrix kennzeichnen, während die Organisationstypen der Fertigung durch geeignete Anordnung der Zeilen und Spalten in der Arbeitseinsatzmatrix erfaßt werden.

Um zur Produktionsfunktion der Unternehmung zu gelangen, muß die Strukturmatrix in die Direktverbrauchsmatrix überführt werden. Deren Elemente ergeben sich aus den Transformationsfunktionen, welche die quantitativen Input-Output-Beziehungen einzelner Teilprozesse abbilden. Als wichtige Klassen kann man e invariablige Transformationsfunktionen (z. B. Leontief-Funktionen) und mehrvariablige Transformationsfunktionen mit gegenseitig unabhängigen Einflußgrößen (z. B. Einflußgrößenrechnung und Pichler-Modelle) sowie interdependenten Einflußgrößen (z.B. Cobb-Douglas-Funktionen und Gut e n b e rg-Verbrauchsfunktionen) unterscheiden. Setzt man für eine zugrunde gelegte betriebliche Produktionsstruktur bestimmte Transformationsfunktionen in das allgemeine Input-Output-Modell ein, so läßt sich die Produktionsfunktion der Unternehmung herleiten. Hierbei können auch Kuppelprodukte berücksichtigt werden, indem man die gemeinsam erzeugten Güter in Haupt- bzw. Nebenprodukte einteilt und alle Gütereinsätze dem Hauptprodukt zurechnet. Jedoch ist zu beachten, daß dann der Wertebereich der Variablen aufgrund des starren Mengenverhältnisses zwischen den Kuppelprodukten begrenzt ist.

Die - Weiterentwicklung des Input-Output-Modells als Produktionsfunktion der Unternehmung kann insbesondere in der Erfassung dyna$\mathrm{m}$ is che $\mathrm{r}$ Beziehungen liegen. Die Beziehungen zwischen den Variablen verschiedener Perioden können über die Lagerbestände und die in den Transformationsfunktionen abzubildenden Zeitdauern der Fertigungsprozesse hergestellt werden. Damit gelingt es, weitere Bestimmungsgrößen der Produktion zu erfassen und den empirischen Gehalt produktionstheoretischer Aussagen zu erhöhen.

\section{$\mathrm{Sum} \mathrm{mary}$}

The article analyses the input-output-system as a general model of microeconomic production processes. It shows that important characteristics of industrial 
production as material combination, flow-shop and job-shop or joint processes can be described by a matrix of production structure. Furthermore the model can be developed for different types of $t r a n s$ formation $\mathrm{f} u \mathrm{nct}$ ions as Leontief function, Cobb-Douglas function or Gutenberg function. On the basis of a special production structure and special transformation functions the production function of the firm is derived.

\section{Literatur}

Adam, Dietrich, (Kostentheorie) Produktions- und Kostentheorie bei Beschäftigungsgradänderungen. Tübingen, Düsseldorf 1974.

A yres, Frank jr., (Matrices) Theory and Problems of Matrices. New York u. a. 1962.

B ohr, Kurt, (Produktionstheorie) Zur Produktionstheorie der Mehrproduktunternehmung. Traditionelle Theorie und Lineare sowie Nichtlineare Programmierung. Köln und Opladen 1967.

B u t t e r w o r t h, J. E. und S i g 1 o c h, B. A., (Input-Output-Model) A Generalized Multi-Stage Input-Output Model and Some Derived Equivalent Systems. In: The Accounting Review (46) 1971, S. 700-716.

$\mathrm{Cz}$ a y k a, L o th a r, (Input-Output-Analyse) Qualitative Input-Output-Analyse. Die Bedeutung der Graphentheorie für die interindustrielle Input-Output-Analyse. Meisenheim am Glan 1972.

Faddejew, D. K. und Faddejewa, W. N., (Algebra) Numerische Methoden der linearen Algebra. München-Wien 1964.

F el th a m, G. A., (Approaches) Some Quantitative Approaches to Planning for Multiproduct Production Systems. In: The Accounting Review (45) 1970, S. $11-26$.

Franke, Reimund, (Betriebsmodelle) Betriebsmodelle. Rechensysteme für Zwecke der kurzfristigen Planung, Kontrolle und Kalkulation. Düsseldorf 1972.

Große-O e tring ha us, Wig a nd, F.,( Fertigungstypologie) Fertigungstypologie unter dem Gesichtspunkt der Fertigungsablaufplanung. Berlin 1974.

Guten berg, Erich, (Produktion) Grundlagen der Betriebswirtschaftslehre. Erster Band: Die Produktion. 21. Aufl., Berlin, Heidelberg, New York 1975.

$\mathrm{He}$ i n e n, E d m u nd, (Kostenlehre) Betriebswirtschaftliche Kostenlehre. Kostentheorie und Kostenentscheidungen. 4. Aufl., Wiesbaden 1974.

$\mathrm{Jacob}$, Herbert, (Produktionsplanung) Produktionsplanung und Kostentheorie. In: Zur Theorie der Unternehmung. Festschrift zum 65. Geburtstag von Erich Gutenberg. Hrsg. von Helmut Koch. Wiesbaden 1962, S. 205-268.

Kilger, Wolfgang, (Produktionstheorie) Produktions- und Kostentheorie. Wiesbaden 1958.

Kloock, Josef, (Input-Output-Modelle) Betriebswirtschaftliche Input-Output-Modelle. Ein Beitrag zur Produktionstheorie. Wiesbaden 1969.

- (Produktionstheorie) Zur gegenwärtigen Diskussion der betriebswirtschaftlichen Produktionstheorie und Kostentheorie. In: Zeitschrift für Betriebswirtschaft (39) 1969, 1. Ergänzungsheft S. 49-82.

- (Input-Output-Analyse) Input-Output-Analyse. In: Handwörterbuch der Betriebswirtschaft. 4. Aufl. Hrsg. von Erwin Grochla und Waldemar Wittmann. Stuttgart 1975, Sp. 1953-1966. 
K n o p, W., (Kostenmodellierung) Probleme und Möglichkeiten der Kostenmodellierung in Chemiebetrieben. In: Chem. Techn. (21) 1969, S. 45-51 und 105-108.

Koopmans, Tjalling C., (Production) Analysis of Productions as an Efficient Combination of Activities. In: Activity Analysis of Production and Allocation. Hrsg. von Tjalling C. Koopmans. New York, London und Sydney 1951, S. 33-97.

Kosiol, Erich, (Organisation) Organisation der Unternehmung. Wiesbaden 1962.

- (Aktionszentrum) Die Unternehmung als wirtschaftliches Aktionszentrum. Einführung in die Betriebswirtschaftslehre. Reinbek 1966.

L a $B$ m a n n, Gert, (Erlösrechnung) Die Kosten- und Erlösrechnung als Instrument der Planung und Kontrolle in Industriebetrieben. Düsseldorf 1968.

L a uenstein, G., (Probleme) Probleme bei der Aufstellung und Anwendung von Matrizenmodellen der Kosten in Chemiebetrieben. Diss. TH f. Chemie Leuna-Merseburg 1965.

L ü cke, Wolf g a ng, (Produktionstheorie) Produktions- und Kostentheorie. 3. Aufl., Würzburg-Wien 1973.

Melle rowicz, Kon rad, (Industrie) Betriebswirtschaftslehre der Industrie. 6. Aufl., Band II, Freiburg i. B. 1968.

Pichler, O., (Anwendung) Anwendung der Matrizenrechnung auf betriebswirtschaftliche Aufgaben. In: Ingenieur-Archiv (21) 1953, S. 119-140.

- (Matrizenrechnung) Anwendung der Matrizenrechnung zur Erfassung von Betriebsabläufen. In: Ingenieur-Archiv (21) 1953, S. 157-175.

- (Betriebskostenüberwachung) Anwendung der Matrizenrechnung bei der Betriebskostenüberwachung. In: Anwendungen der Matrizenrechnung auf wirtschaftliche und statistische Probleme. Von Adolf Adam u.a. Würzburg-Wien 1966, S. 74-111.

Pressmar, Di eter B., (Leistungsanalyse) Kosten- und Leistungsanalyse im Industriebetrieb. Wiesbaden 1971.

REFA, (Arbeitsstudium 1) Methodenlehre des Arbeitsstudiums. Teil 1. Grundlagen. München 1971.

REFA, (Arbeitsstudium 3) Methodenlehre des Arbeitsstudiums. Teil 3. Kostenrechnung, Arbeitsgestaltung. München 1971.

Riebel, P a u 1, (Kuppelproduktion) Die Kuppelproduktion. Betriebs- und Marktprobleme. Köln und Opladen 1955.

- (Erzeugungsverfahren) Industrielle Erzeugungsverfahren in betriebswirtschaftlicher Sicht. Wiesbaden 1963.

$\mathrm{Sch}$ äfer, Erich, (Industriebetrieb) Der Industriebetrieb. Betriebswirtschaftslehre der Industrie auf typologischer Grundlage. Band 1. Köln und Opladen 1969.

Schweitzer, Marcell, (Probleme) Probleme der Ablauforganisation in Unternehmungen. Berlin 1964.

- (Kostenremanenz) Kostenremanenz. In: Handwörterbuch des Rechnungswesens. Hrsg. von Erich Kosiol. Stuttgart 1970, Sp. 967-974.

- (Industriebetriebslehre) Einführung in die Industriebetriebslehre. Berlin, New York 1973.

- (Kostentheorie) Zum gegenwärtigen Stand der linearen Produktions- und Kostentheorie. Arbeitsbericht Nr. 1 der Forschungsabteilung für Industriewirtschaft am Wirtschaftswissenschaftlichen Seminar der Eberhard-KarlsUniversität Tübingen. Tübingen 1976. 
Schweitzer, Marcell und Küpper, Hans-Ulrich, (Produktionstheorie) Produktions- und Kostentheorie der Unternehmung. Reinbek 1974.

$\mathrm{S} \mathrm{chum}$ a n n, J o che n, (Input-Output-Analyse) Input-Output-Analyse. Berlin, Heidelberg, New York 1968.

$S$ t ef f e n, M. und $S$ t e in e cke, V., (Einflußgrößenrechnung) Einflußgrößenrechnung zur Kostenplanung eines Feinstahlwalzwerkes mit Matrizen. In: Stahl und Eisen (82) 1962, S. 155-165.

$\mathrm{S} t$ ef $\mathrm{fe} n, \mathrm{R}$ e in er, (Elementarfaktoren) Analyse industrieller Elementarfaktoren in produktionstheoretischer Sicht. Grundlagen für den Aufbau kurzfristiger Planungsmodelle. Berlin 1973.

Vogel, Fritz, (Strukturbilanzen) Betriebliche Strukturbilanzen und Strukturanalysen. Würzburg-Wien 1969.

Wa rtmann, R., (Erfassung) Rechnerische Erfassung der Vorgänge im Hochofen zur Planung und Steuerung der Betriebsweise sowie der Erzauswahl. In: Stahl und Eisen (83) 1963, S. 1414-1425.

Wit tmann, Waldemar, (Produktionstheorie) Produktionstheorie. Berlin, Heidelberg, New York 1968.

Z s c h o cke, D i e tri ch, (Betriebsökonometrie) Betriebsökonometrie. Stochastische und technologische Aspekte bei der Bildung von Produktionsmodellen und Produktionsstrukturen. Würzburg-Wien 1974.

Dr. Hans-Ulrich Küpper, Wirtschaftswissenschaftliches Seminar, Mohlstr. 36, 7400 Tübingen. 